\title{
Long noncoding RNAs expressed in human hepatic stellate cells form networks with extracellular matrix proteins
}

\author{
Chan Zhou', Samuel R. York', Jennifer Y. Chen'1, Joshua V. Pondick'1, Daniel L. Motola', Raymond T. Chung ${ }^{1}$ \\ and Alan C. Mullen ${ }^{1,2^{*}}$
}

\begin{abstract}
Background: Hepatic fibrosis is the underlying cause of cirrhosis and liver failure in nearly every form of chronic liver disease, and hepatic stellate cells (HSCs) are the primary cell type responsible for fibrosis. Long noncoding RNAs (IncRNAs) are increasingly recognized as regulators of development and disease; however, little is known about their expression in human HSCs and their function in hepatic fibrosis.
\end{abstract}

Methods: We performed RNA sequencing and ab initio assembly of RNA transcripts to define the IncRNAs expressed in human HSC myofibroblasts. We analyzed chromatin immunoprecipitation data and expression data to identify IncRNAs that were regulated by transforming growth factor beta (TGF- $\beta$ ) signaling, associated with super-enhancers and restricted in expression to HSCs compared with 43 human tissues and cell types. Co-expression network analyses were performed to discover functional modules of IncRNAs, and principle component analysis and K-mean clustering were used to compare IncRNA expression in HSCs with other myofibroblast cell types.

Results: We identified over 3600 IncRNAs that are expressed in human HSC myofibroblasts. Many are regulated by TGF- $\beta$, a major fibrotic signal, and form networks with genes encoding key components of the extracellular matrix $(E C M)$, which is the substrate of the fibrotic scar. The IncRNAs directly regulated by TGF- $\beta$ signaling are also enriched at super-enhancers. More than 400 of the IncRNAs identified in HSCs are uniquely expressed in HSCs compared with 43 other human tissues and cell types and HSC myofibroblasts demonstrate different patterns of IncRNA expression compared with myofibroblasts originating from other tissues. Co-expression analyses identified a subset of IncRNAs that are tightly linked to collagen genes and numerous proteins that regulate the ECM during formation of the fibrotic scar. Finally, we identified IncRNAs that are induced during progression of human liver disease.

Conclusions: IncRNAs are likely key contributors to the formation and progression of fibrosis in human liver disease.

\section{Background}

Liver fibrosis occurs as a result of chronic liver injury and, if left unchecked, often proceeds to cirrhosis and liver failure $[1,2]$. Fibrosis develops as the result of accumulation of extracellular matrix (ECM) proteins, including collagen and glycoproteins [3-6], in a process that is driven primarily by transforming growth factor beta (TGF- $\beta$ ) signaling [7, 8]. Hepatic stellate cells (HSCs) are the primary source of the ECM proteins that cause

\footnotetext{
* Correspondence: acmullen@mgh.harvard.edu

${ }^{1}$ Gastrointestinal Unit, Department of Medicine, Massachusetts General Hospital, Harvard Medical School, 55 Fruit Street, Boston, MA 02114, USA ${ }^{2}$ Harvard Stem Cell Institute, Cambridge, MA 02138, USA
}

fibrosis $[9,10]$. In response to liver injury, quiescent HSCs become activated and produce ECM proteins $[9,11,12]$. When the source of liver injury is removed, activated HSCs revert to an inactive phenotype, resulting in reduced ECM protein expression [13, 14]. In chronic liver disease, the continual activation of HSCs results in differentiation into HSC myofibroblasts and constitutive production of ECM proteins [2]. Collagen is the primary component of the fibrotic scar, and TGF- $\beta$ is a key signal that promotes collagen expression in HSC myofibroblasts [15-17].

Differentiation of human HSCs into HSC myofibroblasts occurs in vivo in response to chronic liver injury and this process can be modeled ex vivo by growth of 
HSCs on plastic $[9,11]$. Quiescent HSCs are more buoyant than other liver cells due to the presence of fat droplets and can be isolated by density centrifugation [11]. Culture of quiescent HSCs on plastic results in morphological changes and induction of genes, including ACTA2 (actin, alpha2 smooth muscle), LOX (lysyl oxidase), and LOXL2 (lysyl oxidase like 2), which are characteristic of HSC myofibroblasts [18-21]. Despite an understanding of the protein-coding genes that regulate fibrosis and development of ex vivo tissue culture models to study this process, there are still no effective treatments directed at HSCs to inhibit fibrosis and prevent progression of liver disease.

In recent decades, genome-wide studies have uncovered evidence for extensive transcription outside the regions of DNA that encode proteins [22]. Long noncoding RNA (lncRNA) transcripts are greater than 200 nucleotides (nt) in length and have the same structure as messenger RNAs (mRNAs), including a 5' cap and a polyadenylated 3 ' tail, but do not encode proteins [23]. Over 56,000 lncRNA loci have now been described in human cells [24] and new lncRNAs continue to be identified as new tissues and cell types are analyzed. IncRNAs were originally described as regulators of chromatin [25-27], but as increasing numbers of lncRNAs have been analyzed, it has become clear that they play essential roles in many different cellular processes [28-30]. They are also increasingly recognized as key regulators in mammalian development and disease [30-38], but very little is known about their role in liver fibrosis.

In liver disease, lncRNAs have been studied primarily in relation to cancer. HULC, MALAT-1, TUC338, TUC339, lncRNA-HEIH, MVIH, HOTAIR, lnc-RoR, and HOTTIP have all been associated with higher expression in hepatocellular carcinoma (HCC) compared with normal liver tissue [39-48], while MEG3 is repressed in HCC [41]. Expression of MALAT-1, HOTAIR, and IncRNA-HEIH was also found to be predictive of HCC recurrence [42, 43, 49] and expression of HOTTIP correlates with metastatic HCC burden [46]. HULC can be detected in peripheral blood and TUC339 can be detected in extracellular vesicles, suggesting that each might be able to serve as biomarkers for HCC [39, 50]. Outside of cancer, lnc-LALR1 is induced in mouse models of liver regeneration, where it promotes hepatocyte proliferation [51]. In addition, MEG3 is repressed in models of liver injury and in response to TGF- $\beta$ signaling in the HSC line LX2 [52] and GAS5 promotes p27 expression to inhibit HSC proliferation and activation [53]. The IncRNAs associated with liver disease were discovered by analyzing the expression of candidate lncRNAs [40, 43, 46] or by screening panels of lncRNAs to identify known lncRNAs that are preferentially expressed in HCC [39, 41, 42, 44, 45, 54]. These studies have not defined the full population of
lncRNAs that are expressed in HCC or in liver fibrosis and instead have focused solely on characterizing lncRNAs already described in other cell types.

Many lncRNAs follow cell type-specific patterns of expression [55-57], yet no genome-wide analysis has been performed to identify lncRNAs that are uniquely expressed in HSCs. Thus, we performed RNA-sequencing and ab initio assembly of RNA transcripts to define the lncRNAs expressed in HSC myofibroblasts and those regulated by TGF- $\beta$ signaling. We analyzed proximity to protein-coding genes, chromatin modifications, response to TGF-signaling, cell type-specific patterns of expression, and clustering by co-expression network analyses in order to identify lncRNAs with the potential to regulate hepatic fibrosis.

\section{Methods}

\section{Cell culture}

Fetal HSCs (Sciencell) were grown in Dulbecco's modified Eagle medium (DMEM) with $10 \%$ fetal calf serum (FCS) and $1 \%$ penicillin/streptomycin (P/S). Adult human HSCs were isolated from fresh nonparenchymal liver cells obtained from Triangle Research Laboratories. Nonparenchymal cells were centrifuged at $50 \times g$ for 5 minutes to remove residual hepatocytes. The cells in the supernatant were pelleted at $860 \times g$ for $10 \mathrm{~min}$ before re-suspension in Optiprep (Sigma) diluted to $15 \%$ weight per volume (w/v) with Hanks' balance salt solution without calcium or magnesium. Additional layers of $11.5 \%$ and $8.5 \%$ Optiprep solution were added to the centrifuge tube before centrifugation at $1400 \times g$ for 17 min with no brake. HSCs were enriched at the interface between the 11.5 and $8.5 \%$ layers. These cells were removed and expanded in DMEM with $10 \% \mathrm{FCS}$ and $1 \% \mathrm{P} / \mathrm{S}$. All work with primary human cells was performed with approval of the Massachusetts General Hospital Institutional Review Board (IRB). RNAsequencing analysis was performed on HSC myofibroblasts after seven to eight passages. Induction of the quiescentlike phenotype was performed by culturing HSCs in growth factor reduced Matrigel (BD). Analysis was performed after 3 days in Matrigel for quantitative RT-PCR. HSCs treated with TGF- $\beta$ were grown in serum starvation conditions for $48 \mathrm{~h}$ in media containing DMEM with $0.2 \%$ bovine serum albumin (BSA) and $1 \% \mathrm{P} / \mathrm{S}$. Cells were treated with TGF- $\beta$ (2.5 ng/ml, R\&D systems) for $16 \mathrm{~h}$ prior to harvest.

\section{PCR analysis}

RNA was isolated from HSCs using Trizol Reagent (Life Technologies) followed by DNAse I digestion (Life Technologies). RNA was reversed transcribed with Superscript III (Life Technologies). Quantitative RT-PCR analysis was performed with Taqman primer/probe sets (Life Technologies) using the Bio-Rad CFX384 Real Time System. ACTA2, LOX, LOXL2, and COL1A1 expression 
was normalized to GAPDH. The following Taqman primer/probe sets were used: Hs_00426835, ACTA2; Hs_00942480, LOX; Hs_00158757, LOXL2; Hs_00164004, COL1A1; Hs_02758991, GAPDH.

\section{Microscopy}

Matrigel $(200 \mu \mathrm{l}, \mathrm{BD})$ was distributed across the surface of each well of a 24-well plate and allowed to gel before HSC myofibroblasts were added to the well. Activated HSCs were plated on plastic. After 3 days, the medium was aspirated, and Bodipy 493/503 (Life Technologies) and Hoescht were diluted in media and added to the wells. Bodipy was added at a concentration of $67 \mathrm{pg} / \mu \mathrm{l}$ and Hoescht was added at a concentration of $5 \mathrm{pg} / \mathrm{ul}$. After 45 minutes, the cells were washed twice with Dulbecco's phosphate-buffered saline (DPBS) and imaged with a Nikon A1plus confocal microscope 10× lens. The cells were observed using a pinhole setting of $255.4 \mu \mathrm{m}$. Laser intensity, background level, contrast, and electronic zoom size were collected at the same level for each experiment. Image processing was performed using Adobe Photoshop software.

\section{Preparation of RNA-seq and ChIP-seq libraries}

Total RNA was isolated using Trizol reagent followed by clean up using either the MirVana ${ }^{\circ}$ Isolation Kit (Life Technologies) following instructions for total RNA isolation or re-precipitation after phenol:chloroform and choloroform extractions. RNA quality was assessed via Agilent 2100 Bioanalyzer and samples with RNA integrity numbers (RIN) greater than or equal to 9 were used for library preparation. Isolated RNA was prepared for sequencing according to TruSeq Stranded mRNA Library Prep Kit (Illumina). Chromatin immunoprecipitation (ChIP) was performed using antibodies to detect enrichment of H3K4me3 (07-472, Millipore) and H3K27ac (Ab4729, Abcam) as previously described [58] with the following modifications: $1 \times 10^{7}$ cells were sheared for $5 \mathrm{~min}$ in $1 \mathrm{ml}$ of cell lysis buffer (Covaris) using a Covaris S220 set to a peak wattage of 140 , duty factor $5 \%$ and 200 cycles per burst; immunoprecipitations were performed using $1 \mu \mathrm{g}$ of antibody with $10 \mu \mathrm{l}$ of magnetic beads. ChIP-seq libraries were prepared using the TruSeq ChIP Sample Prep Kit (Illumina). They were sequenced using the Illumina HiSeq 2000 to obtain $100 \times 100$-nt paired-end reads for fetal HSCs and used for ab initio assembly (see below). Illumina HiSeq 4000 was used to obtain 50-nt single-end reads for primary adult HSC myofibroblasts. We used 50-nt single-end reads for ChIP-seq analysis.

\section{Ab initio assembly of transcripts from RNA-seq data}

We mapped each replicate of directional paired-end RNAseq data to the human reference genome (hg19/GRCh37) using TopHat v2.0.10 [59, 60] before assembling transcripts using both Cufflinks [61] and Scripture [62]. The TopHat settings were as follows:

tophat - $p 8$-library-type fr-firststrand -mate-innerdist 50 -mate-std-dev 50 -microexon-search -GTF genes.gtf - $0<$ output-folder $><$ index of reference genome $>$ Reads_end1.fastq Reads_end2.fastq

The reference genes in GTF file format (genes.gtf) were downloaded from the University of California, Santa Cruz (UCSC) genome browser [63]. We then assembled transcripts through the following settings of Cufflinks using TopHat output bam file as input:

cufflinks -p 8 -max-bundle-frags 100000000 -librarytype fr-firststrand -frag-bias-correct - multi-readcorrect $-o<$ output_folder $><$ tophat_output_bam_file $>$

Max-bundle-frags was set to $100,000,000$ such that highly expressed genes would be included in the output.

For analysis in Scripture, we used the following TopHat settings:

\section{tophat - 4 -microexon-search -GTF genes.gtf -o $<$ output_folder $><$ index_of_reference_genome $>$ $<$ Reads_one_end.fastq $>$}

We used Scripture (beta2 version) to assemble transcripts by following the protocol for transcript assembly (http://www.broadinstitute.org/software/scripture/). All transcripts assembled in Cufflinks and/or Scripture were then merged into one list through Cuffmerge [61].

\section{Identification of long noncoding RNAs}

The assembled transcripts were then filtered through the following steps to identify lncRNAs:

1. Removed transcripts that overlapped with annotated protein-coding genes, pseudogenes, rRNAs, tRNAs, small nucleolar RNAs (snoRNAs) and microRNAs on the same strand.

2. Removed transcripts with protein coding potential. The coding potential of each remaining transcript was estimated by HMMER protein domain search $[64,65]$ and CPAT [66] using an alignment-free logistic regression model. The Pfam protein families database (v27.0) was downloaded from EMBL-EBI [67]. Both Pfam-A, containing high-quality and manual curated families, and Pfam-B, containing automatically generated comprehensive protein families, were used in the HMMER domain search. We removed the transcripts matching a protein domain with $p$ value $<1 \mathrm{e}-4$. CPAT uses a logistic 
regression model built with four sequence features: open reading frame size, open reading frame coverage, Fickett TESTCODE statistic, and hexamer usage bias to estimate the coding ability of transcripts. We used 0.364 as the threshold for discriminating noncoding and coding transcripts. This threshold (0.364) was chosen because it gives the highest sensitivity and specificity (0.966 for each) for human data [66] according to the nonparametric two-graph receiver operating characteristic (ROC) curves.

3. Removed remaining transcripts that overlapped on the same strand with any transcripts removed in steps 1 or 2 .

4. Removed remaining transcripts that lacked H3K4me3 occupancy within $1 \mathrm{~kb}$ of their $5^{\prime}$ end.

5. Removed remaining transcripts that were shorter than $200 \mathrm{nt}$ or had low read coverage as defined as less than 0.01 reads per kilobase per million unique mapped reads or less than ten reads per transcript.

\section{ChIP-Seq analysis for HSCs}

ChIP-seq datasets were aligned to the human reference genome (hg19/G37) using Bowtie2 [68]. We performed alignment in end-to-end alignment mode with the settings "bowtie2 -k2 -N1-L32 -end-to-end". We next used the MACS2 [69] callpeak function to compare the mapped bam files of each ChIP to its matched whole cell extract background control. H3K4me3 and H3K27ac peaks were predicted using the following setting: $-q 0.01$-nomodel - shiftsize $=150$. This setting was used because histone marks have an underlying characteristic fixed resolution for nucleosome size and our ChIP-seq only sequenced 50 nt at the 3' end. SMAD3 ChIP-seq data from LX2 cells were downloaded from the Gene Expression Omnibus (GEO; accession GSM934613 and GSM934616) and SMAD3 peaks were called by using the default settings in MACS2. We defined genes as bound by SMAD3 if the site of occupancy was within $10 \mathrm{~kb}$ upstream of the transcription start site (TSS) or within the gene body.

\section{Identification of super-enhancers}

H3K27ac peaks that were called using MACS2 were analyzed using ROSE $[70,71]$ to classify enhancers into typical enhancers and super-enhancers based on H3K27ac signals. The human reference genome (build hg19) and H3K27ac peaks were used as input files. lncRNAs were considered to be associated with super-enhancers or typical enhancers if the enhancer was located within $10 \mathrm{~kb}$ of the lncRNA TSS.

\section{Classification of IncRNAs}

We classified lncRNA loci into four categories according to their genomic locations. An lncRNA was classified as divergent if the TSS of the lncRNA locus was within
$2 \mathrm{~kb}$ of the TSS of a protein-coding gene on the opposite strand. Any remaining lncRNAs that were antisense to a protein-coding gene and overlapped the protein-coding gene by one or more base pairs were classified as natural antisense. Remaining lncRNAs located within a 1000-bp window of a region of H3K27ac occupancy were classified as enhancer-associated. Any remaining lncRNAs that had a TSS greater than $2 \mathrm{~kb}$ from the TSS of the nearest protein-coding gene were classified as intergenic.

\section{Calculation of expression levels for protein-coding genes and IncRNAs}

The expression levels of all protein-coding genes and lncRNA loci, represented in fragments per kilobase of transcript per million fragments mapped (FPKM), were calculated by Cuffdiff (v2.2.1) with the following parameters: "-max-bundle-frags 100000000 -library-type fr-firststrand $-b<h g 19$ reference genome $>-$ multi-readcorrect -no-effective-length-correction -min-isoform-fraction 0 -min-alignment-count". The max-bundle-frags setting was increased from the default parameters so that highly expressed genes would not be excluded.

\section{Differential expression analyses}

To increase the sensitivity in detecting changes in expression of protein-coding and lncRNA genes, two approaches were used to quantify changes in expression: (a) TopHat [59, 72] and Cuffdiff (v2.2.1) [61] and (b) HTSeq [73] and DESeq2 [74]. We took the union of differential expressed lncRNAs found either by Cuffdiff or DESeq2 (adjusted $p<0.05$ ). We used the FPKM values to quantify changes in expression for all figures unless otherwise stated.

\section{Comparison of IncRNAs and protein-coding gene structure}

To compare the number of exons, transcript lengths, and gene lengths between lncRNAs in HSCs and proteincoding genes, the longest isoform for each locus was selected to represent an IncRNA or protein-coding gene. All protein-coding genes expressed in HSCs were used for these comparisons.

\section{Raw RNA-seq data of other human tissues and cell types}

We obtained the raw RNA-seq data for 37 human tissues (see Additional file 1: Table S1 for tissue name and GTEx ID) and dermal fibroblasts from dbGaP [75]. RNA-seq data from the six tier 1 and tier 2 Encyclopedia of DNA Elements (ENCODE) cell lines were downloaded from GEO. GM12878, K562, HeLa-S3, HepG2, and human umbilical vein endothelial cell (HUVEC) data were obtained from GSE26284 and H1 (WA01) human embryonic stem cell data from GSE41009. RNAseq data of pancreatic stellate cells and immortalized 
human induced fibroblast (hiF-T) cells were downloaded from GSE43770 and GSE62777, respectively.

\section{Analysis of IncRNA expression across tissues and cell types}

Three samples were selected from each of 37 distinct human tissues from the Genotype-Tissue Expression (GTEx) project [75] and downloaded from dbGaP. The HSC myofibroblasts analyzed in this study were male, so the two male and one female samples with the highest number of reads were selected for each tissue (Additional file 1: Table S1). Where tissues were male- or femalespecific, all three samples were selected from the same sex. The FPKM values for protein-coding and lncRNA genes were used to quantify changes in expression. The Wilcoxon-Mann-Whitney test was used to identify the lncRNAs overexpressed in HSC myofibroblasts and HSC myofibroblasts treated with TGF- $\beta$ compared with 37 human tissues and six ENCODE cell lines. The dendrogram for clustering the human samples was calculated by the default setting of the $R$ function heatplot (in the gplots and made4 libraries of $R$ ).

RNA-seq data from 35 liver samples from GTEx [75] were downloaded from dbGaP. The pathology reads were obtained from GTEx. Of the 35 liver samples, eight showed normal histology, two showed bridging fibrosis, and two showed cirrhosis (Additional file 2: Table S2). These 12 samples were selected for further analysis. The Z-score was calculated by subtracting the mean expression of an lncRNA for each row from the individual expression level of the IncRNA in a sample and dividing by the standard deviation.

\section{Generation of BigWig files}

Fetal HSC RNA-seq data and the RNA-seq data of six ENCODE cell types are directional, paired-end data and both paired-end reads were mapped independently to the reference genome (hg19) in order to retain strandspecific information for HSC and ENCODE RNA-seq data. In this way the second-strand sequence was mapped to the genome and the reverse complement of the firststrand sequence was mapped to the genome. We then converted the mapped reads with strand information into BigWig files.

The RNA-seq data from GTEx is undirectional pairedend data, so we mapped both end reads into the human reference genome without strand information. BigWig files for ENCODE and GTEx were generated using TopHat to align to the genome with the setting " $-\mathrm{N} 0$ ", which was necessary to exclude a peak in this region that mapped to multiple genomic locations.

We used the " $b d g c m p$ " function of MACS2 to subtract the whole-cell extract background sequencing reads from the ChIP-seq reads using the log-likelihood ratio $(\log L R)$ method, which calculates the $\log 10$ likelihood ratio between the ChIP and whole-cell extract. All ChIP and whole-cell extract background sequencing reads were normalized by their sequencing depths. We then converted the mapped reads into BigWig files.

\section{Co-expression analysis and network construction}

We constructed the co-expression networks for lncRNAs and all protein-coding genes from RefSeq (version of 10 Feb 2014) using the mcxarray program in the Markov Clustering (MCL)-edge network analysis tool [76] (http:// micans.org $/ \mathrm{mcl} /$ ) with Spearman correlation. Gene expression was calculated using Cuffdiff (see the previous section in the "Methods" for details). In this study, we choose 0.7 as the Spearman correlation cutoff in order to balance the number of singletons and the median node degree as recommended by the MCL protocol. To make the co-expression analysis consistent through the entire project, we used the same correlation and threshold to examine if the divergently transcribed coding genes and lncRNAs are co-expressed.

We adopted the MCL algorithm to identify clusters from the constructed large networks. The MCL algorithm is coded in the $m c l$ program [76], which is a fast and scalable unsupervised cluster algorithm for networks based on simulation of stochastic flow in networks. The default granularity "-I 1.4" was set for MCL clustering. Cytoscape v3.1.1 [77] was used to visualize the connected networks and clusters.

For the nucleotide-binding module, all the proteincoding genes annotated in the Gene Ontology (GO) nucleotide binding category in cluster I and their directly co-expressed lncRNAs (linking by one-edge) in cluster I were selected to be displayed. For the extracellular matrix module, all the protein-coding genes annotated in the GO extracellular matrix category in cluster II and their directly co-expressed lncRNAs (linking by oneedge) in cluster II were selected to be displayed.

\section{GO enrichment analyses}

GO enrichment analysis was performed using the proteincoding genes identified in each co-expression cluster (http://david.abcc.ncifcrf.gov/) [78, 79]. All protein-coding genes used in the network construction were used as the background in the GO enrichment analyses.

\section{Statistical analysis}

The $p$ values in this study were calculated by WilcoxonMann-Whitney test, unless otherwise mentioned.

\section{Principal component analysis}

We used pricipal component analysis (PCA) to examine the similarity in expression patterns of lncRNAs among the indicated cell types and to examine the similarity in 
expression patterns of lncRNAs plus protein coding genes between the same cell types. Each replicate for each sample is represented with a vector of the expression levels of the indicated genes. All principal components (PCs) were identified for each replicate through PCA on its expression vector. We then used the k-mean method to cluster the replicates of all samples according to the first three major components. Graphs display the first and second major components (PC1 and PC2) or the first and third major components (PC1 and PC3) in two dimensions.

\section{Identification of enhancer-RNAs}

To identify the enhancer-RNAs (eRNAs), we downloaded the H3K4me3 and H3K4me1 ChIP-seq data for hiF-T cells (GSE62777). We excluded lncRNAs expressed in HSC myofibroblasts that were classified as divergent or natural antisense from eRNA analysis because their histone marks cannot be distinguished from marks of neighboring genes [80]. For the remaining lncRNAs, we selected those that are also expressed in hiF-T cells according to their calls by Cuffdiff. Next, we mapped the raw H3K4me3 and H3K4me1 reads to the TSS regions of each lncRNA (defined as within \pm 500 bp of the TSS) and then calculated the H3K4me1/H3K4me3 ratio for each lncRNA. A ratio of $>1.2$ was used to define eRNAs, as previously described [80].

\section{Data access}

RNA-seq and ChIP-seq data produced for this study are available in the GEO (accession GSE68108).

\section{Results}

\section{De novo identification of IncRNAs in human HSCs}

We first established that primary human fetal HSC myofibroblasts share characteristics described for HSCs. These cells were chosen for initial analysis as they could be expanded more easily for genome-wide sequencing. Culture in Matrigel repressed ACTA2, LOX, and LOXL2 expression and induced accumulation of lipid droplets characteristic of the reversion to an inactive HSC phenotype in Matrigel (Fig. 1a, b) [81, 82]. These HSCs also showed induced collagen expression in response to TGF- $\beta$ signaling (Fig. 1c) [15]. We then performed massively parallel sequencing of polyadenylated RNAs (RNA-seq) to identify lncRNAs that are expressed in human HSC myofibroblasts. Directional libraries were prepared for pairedend sequencing so that overlapping sense and antisense transcripts could be distinguished from one another during analysis. We then established a computational pipeline for ab initio construction of lncRNA transcripts (Fig. 1d) $[58,62,83]$. RNA-seq reads were first aligned to the genome with TopHat [59,60]. Cufflinks [61] and Scripture [62] were each used to assemble the aligned reads into transcripts, which were then merged by Cuffmerge $[60,61]$. All assembled transcripts that overlapped on the same strand with protein-coding genes in RefSeq [84] or the UCSC database [85] were removed, as well as those overlapping with rRNAs, tRNAs, microRNAs and snoRNAs annotated in RefSeq. The remaining transcripts were analyzed by a HMMER profile search of protein domains $[64,65]$ and CPAT software with a logistic regression model [66] to assess their protein-coding potential (Additional file 3: Figure S1). Transcripts that had low protein-coding potential as predicted by both HMMER and CPAT were considered to be noncoding RNAs. We next performed ChIP-seq using an antibody against the chromatin mark H3K4me3 to identify sites of transcription initiation [86]. All RNA transcripts whose 5' ends were greater than $1 \mathrm{~kb}$ from an $\mathrm{H} 3 \mathrm{~K} 4 \mathrm{me} 3$ site were removed because the absence of a proximal transcription initiation mark suggested that the assembled RNA transcripts might be incomplete. All transcripts less than $200 \mathrm{nt}$ in length and below expression thresholds were removed (see "Methods" for additional details). We identified and assembled 7189 lncRNA transcripts into 2808 lncRNA loci (Additional file 4: Table S3).

Two lncRNAs identified with this pipeline are shown in Fig. 1e. $\ln c R N A-054349$ (top) is an example of a threeexon IncRNA that was not previously annotated and EPHA5-AS1 is an example of a lncRNA for which a single isoform was previously annotated. For both lncRNAs, H3K4me3 occupancy is shown in black (top) and the RNA-seq reads supporting the lncRNAs are shown in red (sense). The genomic structure of each lncRNA is indicated below the tracks in red, with arrows indicating the direction of transcription. EPHA5-AS1 is divergently transcribed from EPHA5 and the RNA-seq reads supporting EPHA5 transcripts are indicated in blue (antisense).

\section{Genomic characterization of IncRNAs}

We next assessed the distribution of lncRNAs across the genome. Over $65 \%$ of HSC lncRNAs were divergently transcribed from the promoter regions of protein-coding genes. Four percent of the total identified lncRNAs were antisense to coding genes (natural antisense), $10 \%$ were located at enhancers as defined by the presence of $\mathrm{H} 3 \mathrm{~K} 27 \mathrm{ac}$ [87], and $15 \%$ originated from intergenic regions away from coding genes (Fig. 2a). Plotting the location of the transcriptional start site (TSS) of each lncRNA relative to the TSS for the closest proteincoding gene showed that the vast majority of lncRNAs are transcribed antisense to protein-coding genes and originate near the promoter of these genes (Fig. 2b), with the TSS of the lncRNAs located a mean distance of $135 \mathrm{nt}$ upstream of the TSS of the paired protein-coding genes. We also found that lncRNAs in HSCs tend to be expressed at lower levels than protein-coding genes. 
a

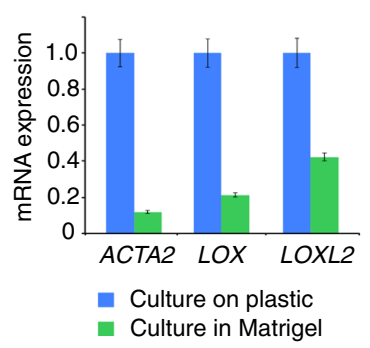

b

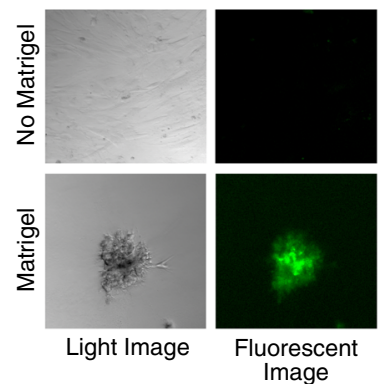

C

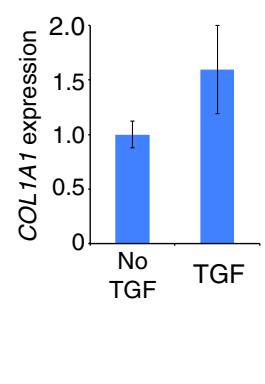

d

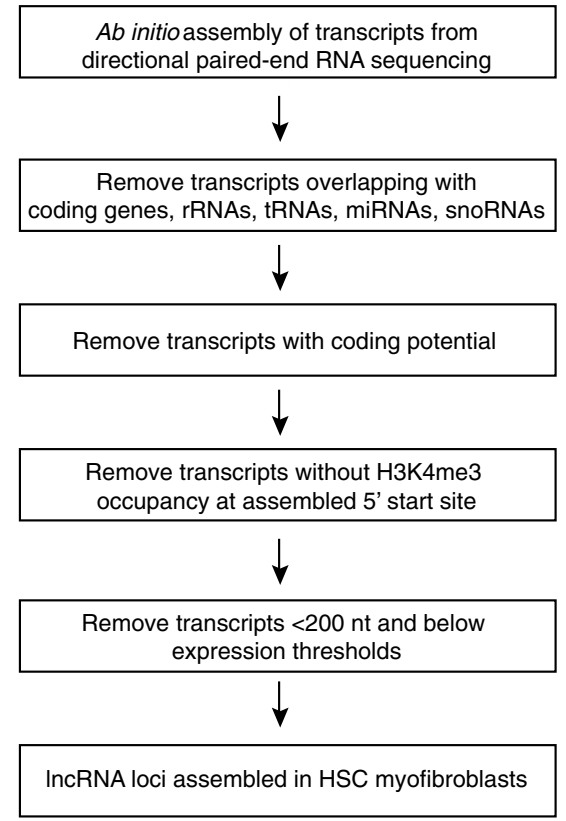

e
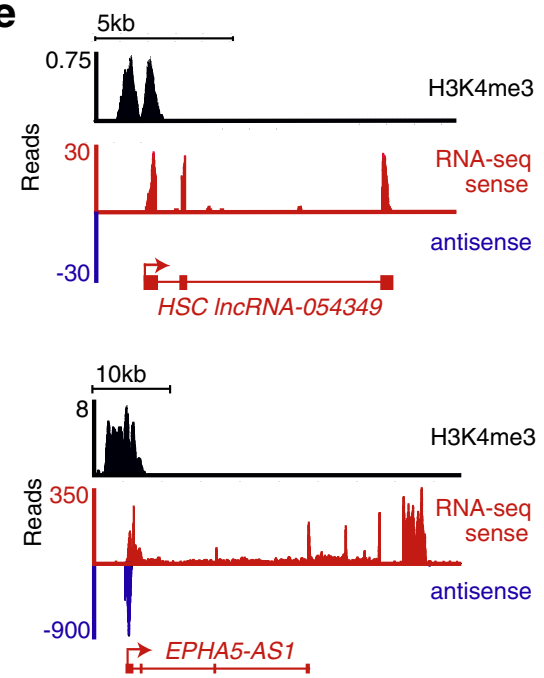

RefSeq "

Gene

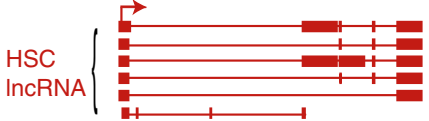

Fig. 1 Identification of IncRNAs in human HSC myofibroblasts. a ACTA2, LOX, and LOXL2 mRNA levels were quantified in HSC myofibroblasts cultured on plastic (blue) and compared with mRNA levels in HSC myofibroblasts that were cultured in Matrigel for 3 days to induce reversion to an inactive phenotype (green). Samples were normalized using GAPDH, and expression of each gene in HSC myofibroblasts was set to 1. Error bars represent standard deviation. This experiment is representative of three biological replicates. b Culture in Matrigel leads to accumulation of fat droplets characteristic of HSCs that have reverted to an inactive phenotype. Microscopy images are shown for HSC myofibroblasts cultured without (No Matrigel) and with Matrigel for 3 days. Light microscopy images (left) show that the cells grown in Matrigel become rounded and clump together. Bodipy staining for neutral fat (green fluorescence, right) shows accumulation of lipid droplets in HSCs cultured in Matrigel. Images are shown at 10X magnification. c COLIA1 mRNA levels were quantified in HSC myofibroblasts cultured in $0.2 \%$ bovine serum albumin and after treatment with TGF- $\beta$ for 16 h. $\mathbf{d}$ Pipeline for identification of IncRNAs (see the "Methods" for details). miRNA microRNA. e Examples of IncRNAs identified in HSC myofibroblasts. IncRNA-054349 is a novel IncRNA discovered in HSCs (top) and EPHA5-ASI is an IncRNA expressed in HSCs that is expressed antisense to EPHA5 (blue). For each IncRNA track, H3K4me3 enrichment (log likelihood ratio (LogLR) value normalized by subtracting the background) marks the site of transcription initiation (black, top). RNA-seq reads supporting IncRNA transcripts are shown in red (RNA-sea sense). Antisense transcripts are shown in blue. The genomic structure for each gene is shown below the RNA-seq tracks. IncRNAs are shown in red and protein-coding genes are shown in blue. Boxes indicate exons, lines indicate introns, and arrows indicate the start and direction of transcription. For EPHA5-AS1, the annotated IncRNA and protein-coding gene are shown below the RNA-seq tracks. The multiple isoforms of EPHA5-AS1 that are identified in HSC myofibroblasts (labeled as HSC InCRNA) are shown at the bottom. Novel IncRNAs identified in this study are named with the prefix "InCRNA" followed by the locus number assigned during assembly

Analysis of the expression level of lncRNAs and their divergently transcribed mRNAs showed that lncRNAs are expressed at approximately tenfold lower levels than protein-coding genes (Fig. 2c; $p<2.2 \mathrm{e}-16$ ). These findings are consistent with the description of lncRNAs in other cell types $[55,56,58,88]$.

Most of the identified lncRNA transcripts ( $>55 \%$ ) are single exon transcripts whereas only $4 \%$ of 
a

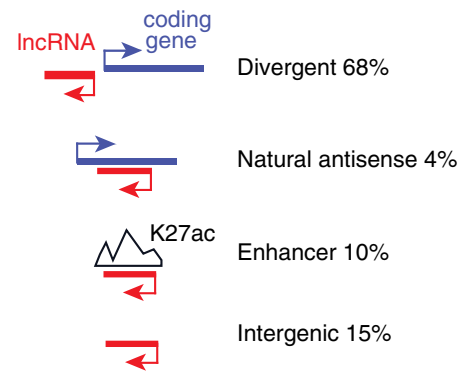

C

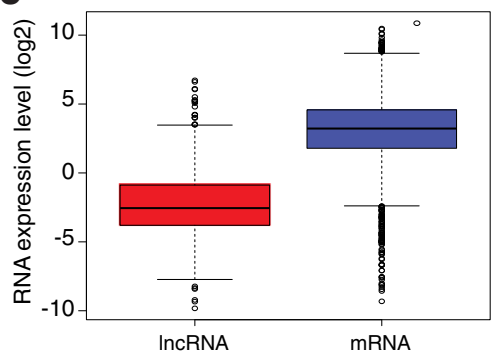

e

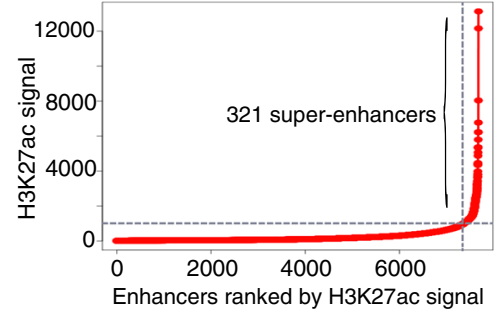

h

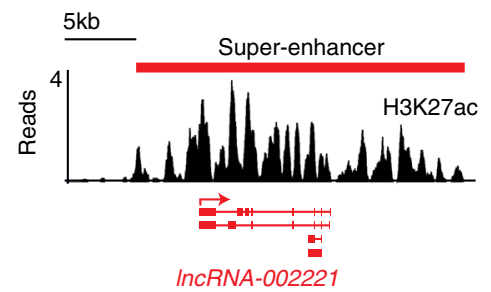

b

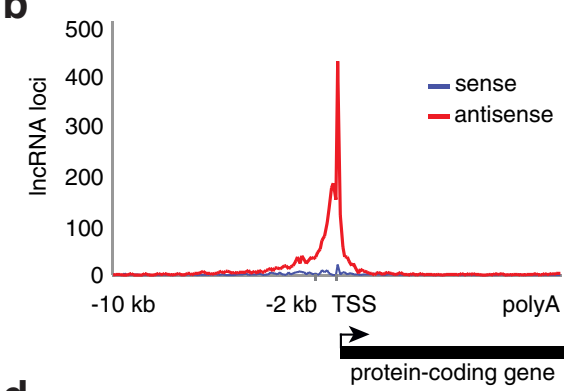

d

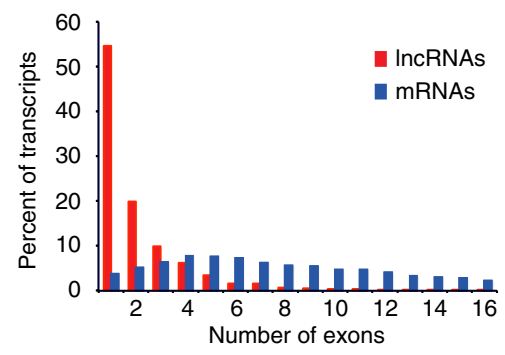

f
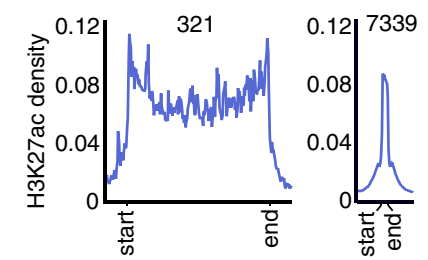

g

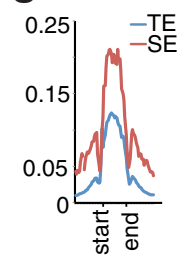

Super-enhancers

Typical

enhancers

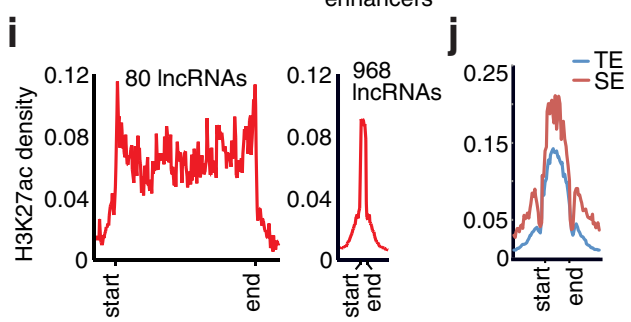

Fig. 2 (See legend on next page.) 


\section{(See figure on previous page.)}

Fig. 2 Genomic and expression features of HSC IncRNAs. a Classification of IncRNA loci identified in HSC myofibroblasts. Divergent IncRNAs are defined as having a transcription start site (TSS) within $2 \mathrm{~kb}$ of the TSS of a protein-coding gene and being transcribed from the strand antisense to the protein-coding gene. Natural antisense IncRNAs overlap the coding gene by at least one base pair. Enhancer-associated IncRNAs are located within $1 \mathrm{~kb}$ of an enhancer as defined by H3K27ac. Intergenic IncRNAs have a TSS greater than $2 \mathrm{~kb}$ from the TSS of the nearest protein-coding gene and are not contained in any of the other categories. IncRNAs are indicated in red and protein-coding genes are indicated in blue. Arrows show the start and direction of transcription. H3K72ac (K27ac) peaks mark enhancers. The abundance of each class of IncRNA is displayed on the right. $\mathbf{b}$ Distribution of the TSS of IncRNAs relative to the TSS of their nearest protein-coding gene. All protein-coding genes were normalized to equal length on the $x$-axis (black rectangle), and $10 \mathrm{~kb}$ of genomic sequence upstream of the TSS of each protein-coding gene is shown. The location of the TSS of each IncRNA was plotted relative to the TSS of the nearest protein-coding gene. IncRNAs that are antisense to protein-coding genes are indicated in red and IncRNAs that are sense to protein-coding genes are indicated in blue. The red peak near the protein-coding TSS indicates that the majority of IncRNAs are located within $2 \mathrm{~kb}$ of protein-coding genes and transcribed antisense to protein-coding genes. $\mathbf{c}$ IncRNAs (red) are expressed about tenfold lower than their divergent protein-coding genes (blue). The expression levels (log2 transformed reads per fragment per million mapped reads (FPKM)) are indicated on the $y$-axis. The horizontal black line indicates the mean and open circles indicate outliers. $\mathbf{d}$ IncRNAs contain fewer exons than protein-coding genes. The distribution of exons in IncRNA (red) and mRNA (blue) transcripts. e Identification of super-enhancers in HSC myofibroblasts. Enhancers were defined by H3K27ac occupancy and ranked from left to right (x-axis) by the total reads of H3K27ac mapped to each enhancer (H3K27ac signal, $y$-axis). We identified 321 super-enhancers from a total of 7660 enhancers [70]. f Super-enhancers show broad domains of occupancy compared with typical enhancers. Metagenes represent the mean H3K27ac density (in reads per million unique mapped reads per base pair) across super-enhancers (left) and typical enhancers (right). The metagenes are centered on the enhancer region for each plot and display $3 \mathrm{~kb}$ of sequence flanking each enhancer. The median size of a super-enhancer is $18,807 \mathrm{bp}$ and the median size of a typical enhancer is $678 \mathrm{bp}$. The plots are scaled ( $x$-axis) to reflect the median size of the two classes of enhancers. The increase of signal at the boundary is characteristic of super-enhancers [71] because the boundary represents the H3K27ac peaks at the edges of each super-enhancer. Thus, the peaks at the boundary tend to be aligned with each other while peaks away from the boundaries are distributed more equally. $\mathbf{g ~ H 3 K 2 7 a c ~ p e a k s ~ a r e ~ e n r i c h e d ~ a t ~ s u p e r - e n h a n c e r s . ~ M e t a g e n e s ~ r e p r e s e n t ~ t h e ~ m e a n ~ H 3 K 2 7 a c ~ d e n s i t y ~}$ across the major peak of super-enhancers (SE) and typical enhancers (TE). $\mathbf{h}$ Example of an IncRNA associated with a super-enhancer. H3K27ac occupancy (normalized logLR value, y-axis) is shown surrounding IncRNA-002221 (red). The domain of the super-enhancer is indicated by a red rectangle. i Super-enhancers were found to be associated with 80 IncRNAs. The median size of super-enhancers associated with IncRNAs was $18,699 \mathrm{bp}$ and the median size of typical enhancers associated with IncRNAs was $1032 \mathrm{bp}$. We located 968 IncRNAs within $10 \mathrm{~kb}$ of a typical enhancer. The enhancers were plotted as described in $\mathbf{f}$. $\mathbf{j}$ H3K27ac peaks are enriched at super-enhancers associated with IncRNAs. Metagenes represent H3K27ac density across the major peak of super-enhancers and typical enhancers associated with IncRNAs

protein-coding genes expressed in HSC myofibroblasts are encoded by single exons (Fig. 2d). HSC lncRNAs also tend to have shorter genes and shorter transcripts than protein-coding genes (Additional file 3: Figure S2) [88].

Super-enhancers are large domains of active enhancers associated with genes that control cell identity [70, 71]. To identify lncRNAs that may regulate key facets of HSC identity and function, we asked if lncRNAs are associated with super-enhancers in HSCs. We performed ChIP-seq to identify regions of chromatin containing the histone modification $\mathrm{H} 3 \mathrm{~K} 27 \mathrm{ac}$, which is enriched at active enhancers [87]. We identified 7339 typical enhancers and 321 super-enhancers (Additional file 5: Table S4) according to their H3K27ac signal strengths (Fig. 2e). These super-enhancers are an order of magnitude larger than typical enhancers in genomic coverage (Fig. 2f) and have larger peaks of H3K27ac enrichment than typical enhancers (Fig. 2g). Super-enhancers are also associated with lncRNAs identified in HSCs; for example,

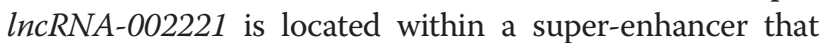
spans greater than $20 \mathrm{~kb}$ (Fig. 2h). Eighty lncRNAs discovered in HSC myofibroblasts were found to be associated with super-enhancers. H3K27ac signal at these superenhancers associated with lncRNAs also shows increased genomic coverage (Fig. 2i) and larger peaks than typical enhancers (Fig. 2j).

\section{TGF- $\beta$ signaling directly regulates IncRNA expression in HSC myofibroblasts}

TGF- $\beta$ is a key regulator of fibrosis in liver disease [15-17] and we next asked if TGF- $\beta$ signaling regulates expression of lncRNAs in HSC myofibroblasts. HSC myofibroblasts were serum starved for $48 \mathrm{~h}$ to remove exogenous TGF- $\beta$ from the media, followed by treatment with TGF- $\beta$ for $16 \mathrm{~h}$ (Fig. 3a). RNA was harvested from HSC myofibroblasts that were treated with TGF- $\beta$ after serum starvation and from HSC myofibroblasts that remained in serum starvation conditions. We performed RNA-seq analysis to confirm that TGF- $\beta$ treated HSCs respond to TGF- $\beta$ signaling (Additional file 3: Figure S3a) and then used our computational pipeline (Fig. 1d) to assemble the lncRNAs expressed during serum starvation (low TGF- $\beta$ ) and after TGF- $\beta$ treatment. Wer assembled 2078 lncRNA loci in serum-starved HSC myofibroblasts and 1759 lncRNA loci after TGF- $\beta$ treatment (Additional file 3: Figure S3b).

The lncRNA transcripts defined in HSC myofibroblasts, serum-starved HSC myofibroblasts, and TGF- $\beta$-treated HSC myofibroblasts were combined using Cuffcompare $[60,61]$ to define a total of 16,299 lncRNA transcripts expressed in at least one condition. These transcripts were classified into 3692 lncRNA loci and represent all the lncRNA loci detected in human HSCs (Additional file 6: Table S5 and Additional file 7: Table S6). Many of these 
a

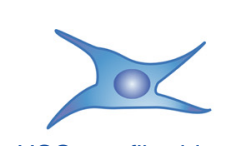

HSC myofibroblast
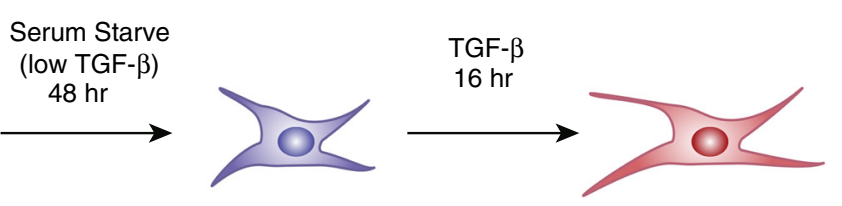

b

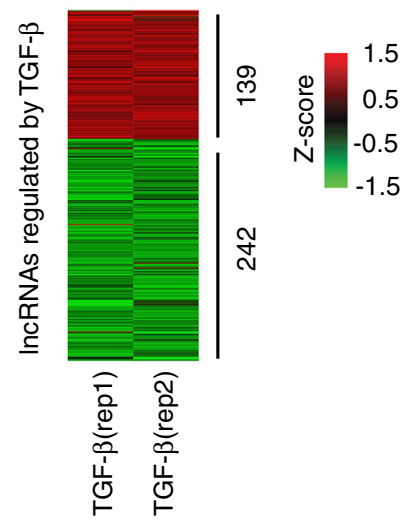

d

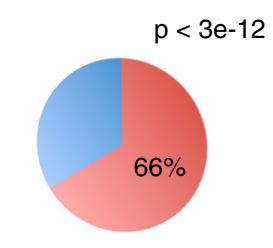

Induced by TGF- $\beta$

SMAD3 occupancy

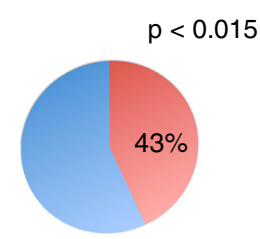

Repressed by TGF- $\beta$

No SMAD3
C

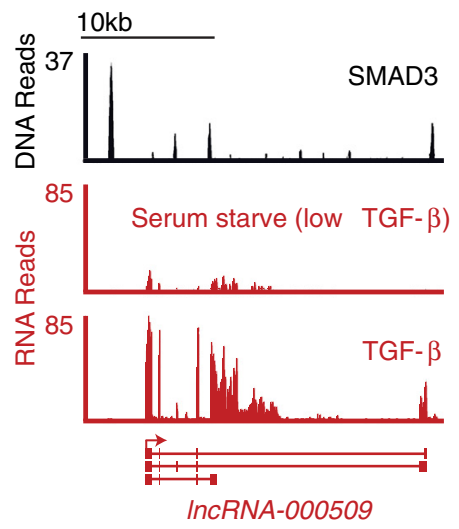

e

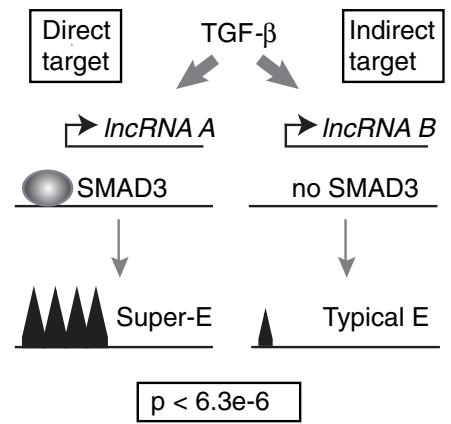

Fig. 3 Regulation of HSC IncRNAs. a Conditions for HSC IncRNA analysis. HSC myofibroblasts were cultured for $48 \mathrm{~h}$ in $0.2 \%$ bovine serum albumin to remove exogenous TGF- $\beta$ from the media (low TGF- $\beta$ ) and then treated with TGF- $\beta$ for $16 \mathrm{~h}$ to define IncRNAs regulated by TGF- $\beta$ signaling. $\mathbf{b}$ IncRNA expression in response to TGF- $\beta$ signaling. The heatmap shows normalized expression of IncRNAs that were induced (red) or repressed (green) in response to TGF- $\beta$ signaling. The Z-score is shown on the right and was calculated from expression of IncRNAs in two replicates of low TGF- $\beta$ conditions (not shown) and two replicates of TGF- $\beta$ treatment (shown). Each row represents one IncRNA locus and IncRNA loci are ranked by $p$ value such that the smallest $p$ value of induced IncRNAs is at the top and the smallest $p$ value of repressed IncRNAs is at the bottom. c The gene encoding IncRNA-000509 is occupied by SMAD3 and induced in response to TGF- $\beta$ signaling. SMAD3 occupancy [90] is shown at the top. RNA-seq reads (red) are displayed for HSCs in low TGF- $\beta$ and after $16 \mathrm{~h}$ of TGF- $\beta$ signaling. The structure and direction of IncRNA-000509 is shown below. $\mathbf{d}$ IncRNAs that are regulated by TGF- $\beta$ signaling tend to be occupied by SMAD3. The fraction of IncRNA genes that are induced (left) and repressed (right) by TGF- $\beta$ and are occupied by SMAD3. Genes that are regulated in response to TGF- $\beta$ signaling and occupied by SMAD3 are considered direct targets of TGF- $\beta$ signaling. e IncRNAs directly targeted by TGF- $\beta$ signaling tend to be associated with super-enhancers. IncRNA genes that change in expression in response to TGF- $\beta$ signaling and are occupied by SMAD3 (Direct target) are associated with super-enhancers (Super-E), while IncRNAs that change in expression in response to TGF- $\beta$ signaling and are not directly occupied by SMAD3 (Indirect target) are associated with typical enhancers (Typical E)

lncRNAs are novel, including $40 \%$ that do not overlap with a single nucleotide of human lncRNAs annotated in existing databases (Additional file 3: Figure S3c).

We identified 139 lncRNA loci that were induced and 242 lncRNA loci that were repressed with activation of
TGF- $\beta$ signaling (Fig. 3b; $p<0.05$ ). The transcription factors SMAD2 and SMAD3 are activated by TGF- $\beta$ signaling to mediate the transcriptional effects of the canonical TGF- $\beta$ signaling pathway [89] and SMAD3 appears to play the dominant role in HSC myofibroblast 
activation [12]. To determine the direct targets of TGF- $\beta$ signaling, we identified the lncRNAs that were regulated by TGF- $\beta$ and occupied by SMAD3. For example, $\operatorname{lncRNA-000509}$ is occupied by SMAD3 and shows a tenfold induction between serum starvation (low TGF- $\beta$ ) and induction of TGF- $\beta$ signaling (Fig. 3c). Sixty-six percent of lncRNAs induced by TGF- $\beta(p<3 e-12)$ and $43 \%$ of lncRNAs repressed by TGF- $\beta(p<0.015)$ were found to be occupied by SMAD3 in the human HSC line LX2, following TGF- $\beta$ treatment [90] (Fig. 3d). This analysis identified 91 lncRNAs that are directly induced by TGF- $\beta$ signaling and 104 lncRNAs that are directly repressed by TGF- $\beta$ signaling, with a mean induction and repression of twofold (Additional file 8: Table S7).

TGF- $\beta$ signaling targets cell type-specific enhancers bound by master transcription factors [91] and superenhancers regulate genes that define cell identity [70, 71]. We also found that lncRNAs directly targeted by TGF- $\beta$ signaling (regulated by TGF- $\beta$ and occupied by SMAD3) are more likely to be associated with super-enhancers in HSCs than lncRNAs indirectly targeted by TGF- $\beta$ signaling (Fig. 3e; $p<6.3 \mathrm{e}-6$ ), suggesting that these lncRNAs may also play a key role in cell identity. Coding genes directly targeted by TGF- $\beta$ signaling are also more likely to be associated with super-enhancers than indirectly targeted genes $(p<5.9 \mathrm{e}-14)$. However, IncRNAs that are directly targeted by TGF- $\beta$ signaling are more likely to be regulated by super-enhancers than coding genes directly targeted by TGF- $\beta(p<1.6 \mathrm{e}-13)$.

\section{IncRNAs enriched in HSC myofibroblasts}

lncRNAs appear to be under weaker evolutionary constraints than protein-coding genes, resulting in more cell type-specific patterns of expression compared with coding genes [55-57]. While approximately $40 \%$ of the 3692 lncRNA loci identified in HSCs were not described in other IncRNA databases (Additional file 3: Figure S3c), it is unclear if this result means that these lncRNAs are unique to HSCs or that many of these IncRNAs are expressed in other cell types but have not been described. To define the lncRNAs specific to HSCs, we examined the expression patterns of IncRNAs identified in HSC myofibroblasts and HSC myofibroblasts treated with TGF- $\beta$ compared with 37 other human tissues [75] and the six tier 1 and tier 2 cell lines from the ENCODE project [92]. Analyses revealed that more than 400 lncRNAs are significantly enriched in HSCs compared with the 43 other tissues and cell types analyzed (Fig. $4 ; p<0.05$;

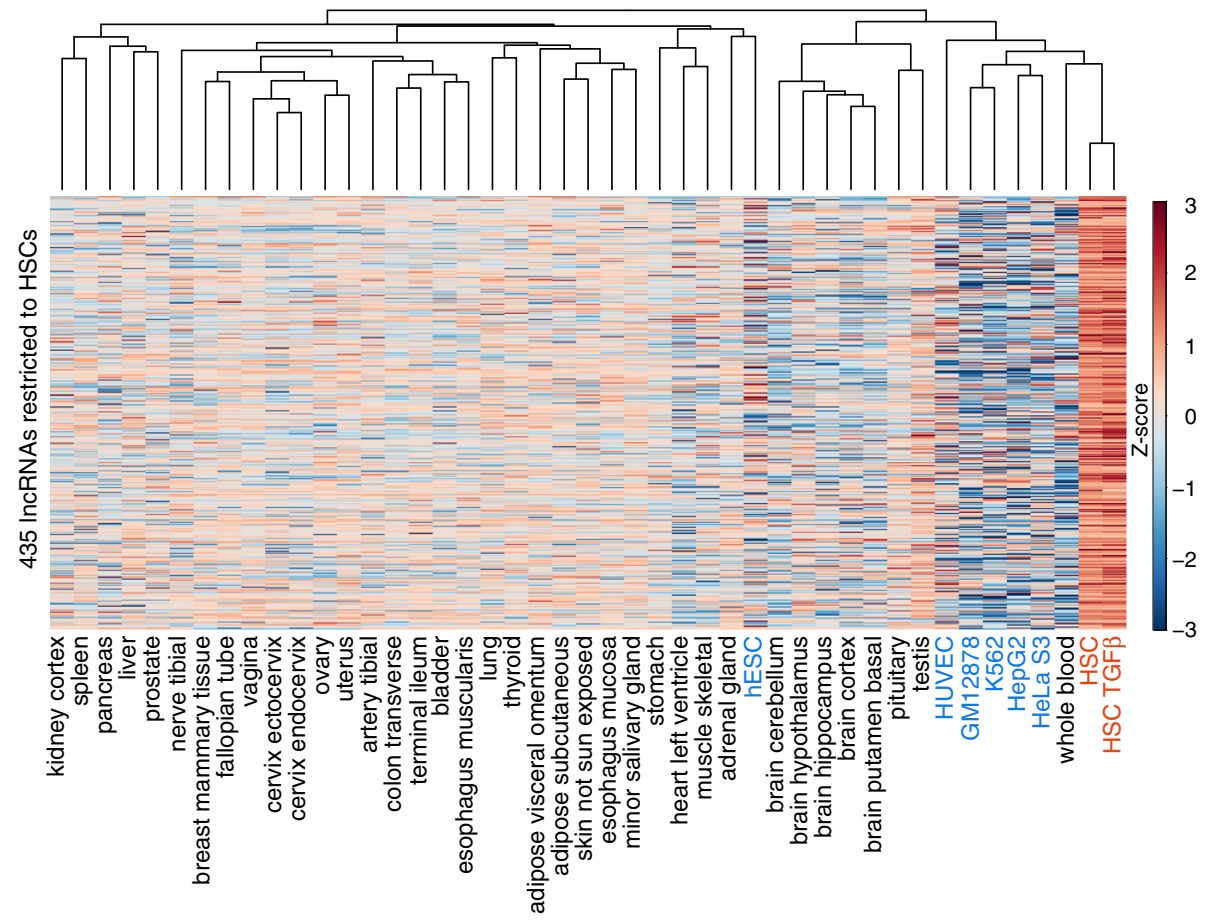

Fig. 4 Expression of HSC-specific IncRNAs. The normalized expression levels are shown for 435 IncRNAs ( $y$-axis) that are enriched in HSCs compared with other cell types and tissues. RNA-seq data from HSC myofibroblasts and HSC myofibroblasts treated with TGF- $\beta$ (red) were compared with 37 normal tissues (black text) [75] and six ENCODE cell lines (blue text) [92]. The dendrogram at the top indicates hierarchical clustering. Each row represents one IncRNA locus. Red shading indicates induction and blue shading indicates repression relative to the levels of each IncRNA across all tissues. The Z-score is shown on the right. IncRNAs were considered HSC-specific if they were enriched in HSCs compared with other tissues with a $p$ value of $<0.05$ (Wilcox-Mann-Whitney test) 
Additional file 9: Table S8). This enrichment is observed relative to primary tissue, including liver, and six cell lines, including the hepatocellular carcinoma cell line HepG2.

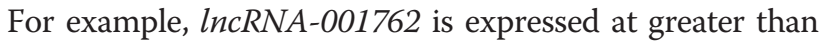
sixfold higher levels in HSC myofibroblasts treated with TGF- $\beta$ compared with the highest level of expression in 43 other tissues and cell lines (Fig. 5a). The FPKM for

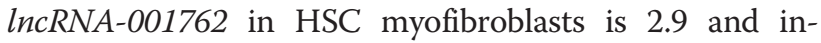
creases to 5.4 with TGF- $\beta$ treatment, whereas the mean in all other samples is 0.13 with maximum expression of 0.85 in human umbilical vein endothelial cells (HUVECs). The restriction in expression to HSCs is further illustrated by visualizing the RNA-seq reads mapped to IncRNA001762 in the HUVEC cell line and testis (Fig. 5b). These examples were chosen because they are the cell line and primary tissue with the next highest expression of lncRNA-001762 compared with HSC myofibroblasts. Whole liver tissue and the HepG2 cells express even lower levels of $\ln R$ RNA-001762, which suggests that this lncRNA is likely restricted in expression to HSCs in the liver and is not induced in hepatocellular carcinoma.

IncRNAs expressed in HSCs are enriched in ECM networks Co-expression network analyses have been used widely to predict the functions of unknown coding and noncoding genes [93-100] based on the expectation that genes with similar expression patterns across multiple tissues, cell types, or conditions share similar functions or are involved in related biological processes [101, 102]. Therefore, we conducted co-expression network analyses across the 37 primary human tissues, six ENCODE cell lines, and the HSCs described in this study using expression profiles for all annotated genes expressed in at least one tissue or cell type and the lncRNAs identified in this study.

We identified 169 subnetworks containing at least two nodes where each node represents a protein-coding gene

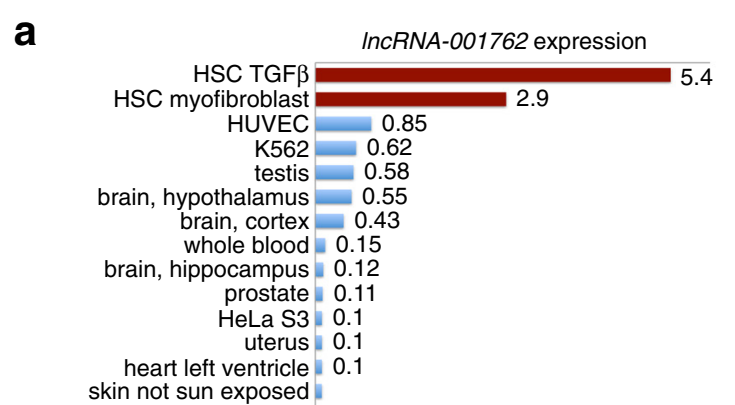

b
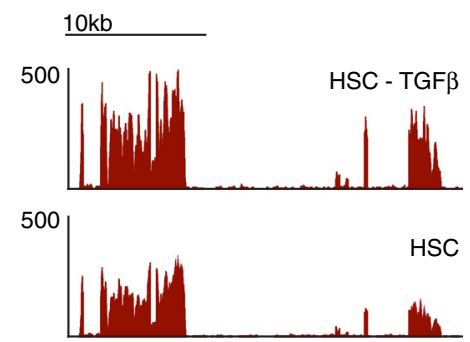
vagina

adipose, visceral omentum

brain, putamen basal

thyroid
thysal thyroid

GM12878
cervix, ectocervix ovary

pancreas

fallopian tube

brain, cerebellum adipose subcutaneous adipose subcutaneous
sm intestine, terminal ileum
liver
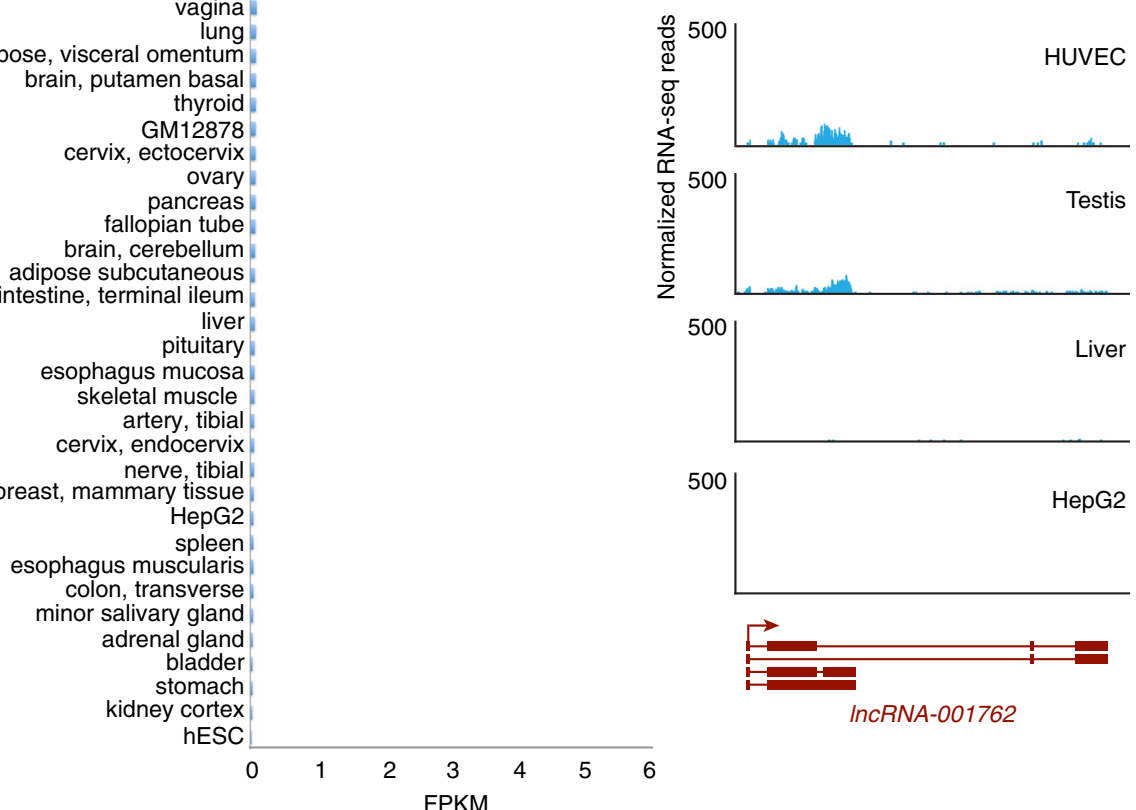
FPKM

Fig. 5 IncRNA-001762 is restricted in expression to HSCs. a Expression of IncRNA-001762 was quantified by FPKM in each cell type (y-axis). The highest level of expression was found in HSCs treated with TGF- $\beta$. $\mathbf{b}$ RNA-seq reads $(y$-axis) were normalized and displayed across the gene encoding IncRNA-001762. Expression was compared with the cell line (HUVEC) and tissue type (Testis) that show the highest levels of IncRNA001762 expression outside HSCs. Expression was also visualized in primary liver tissue and the hepatocellular carcinoma line HepG2. The structure and direction of transcription for InCRNA-001762 is shown below 
or an IncRNA and the edge between two nodes indicates that the two genes are co-expressed with a Spearmancorrelation coefficient $>0.7(p<4 \mathrm{e}-7$; Fig. 6a). Most of the identified subnetworks were small and only five contained more than five nodes. To identify the subnetworks that would provide the most information about lncRNAs acting in HSCs, each subnetwork was scanned for HSC identity lncRNAs that were defined as lncRNAs proximal to super-enhancers, occupied by SMAD3, and restricted in expression to HSCs (Additional file 10: Table S9). Thirty-three lncRNAs met these criteria and 21 were contained in subnetwork A. HSC identity lncRNAs were not found in any of the other subnetworks. Subnetwork A contains 18,002 nodes and was decomposed to identify smaller clusters that may provide further insight into the function of IncRNA genes. Nine clusters were identified based on their connection structure and correlation value (see "Methods" for details) and three clusters contained more than one HSC identity lncRNA. Cluster I contained 3950 nodes, including two identity lncRNAs and 332 lncRNAs expressed in HSCs. Cluster II contained 1865 nodes, including nine identity lncRNAs and 314 lncRNAs expressed in HSCs. Cluster IV contained 48 nodes, including two identity lncRNAs and seven lncRNAs in HSCs.

We then performed gene ontogeny (GO) enrichment analysis on clusters I, II, and IV to identify cellular processes or functions that were key components of each cluster. Cluster I was heavily enriched in nucleotide binding (Fig. 6b), cluster II in ECM (Fig. 6c) and cluster IV in DNA binding factors (Fig. 6d). We visualized cluster I to show the size of the network and the relative composition of mRNA and lncRNA genes by mapping all protein-coding genes from cluster I that were contained in the nucleotide-binding category and all the lncRNAs in cluster I within one edge of these proteincoding genes. This nucleotide-binding functional module is composed of 521 genes encoding mRNA (blue) and 262 genes encoding lncRNAs (red) (Fig. 6e). We visualized the ECM functional module in cluster II by mapping all protein-coding genes from cluster II that were contained in the ECM category and all the lncRNAs in cluster II within one edge of these protein-coding genes. This ECM module is more enriched for lncRNAs, being composed of 110 genes encoding mRNAs and 211 genes encoding lncRNAs (Fig. 6f). We visualized cluster IV by showing all protein-coding genes and lncRNA genes in that cluster. Cluster IV is enriched in HOX transcription factor genes (dark green) and known lncRNA genes associated with HOX loci (light green) (Fig. 6g). Lists of individual genes encoding mRNAs and lncRNAs and the co-expression pairs related to each GO category (nucleotide binding in cluster I, ECM in cluster II and Cluster IV) are included in Additional file 11: Table S10.
The most significant finding from this analysis is that a module of genes encoding mRNAs and lncRNAs is highly associated with the ECM, whose production by HSC myofibroblasts is the primary cause of liver fibrosis and progression to liver failure in chronic liver disease $[9,10]$. To exclude the possibility that the presence of protein-coding genes and lncRNAs highly enriched in HSCs skewed the co-expression network analysis, we repeated the analysis without the HSC data. This analysis also yielded the ECM module within cluster II, demonstrating that this is a robust functional module even in the absence of HSC expression data (Additional file 3: Figure S6).

Twelve HSC identity lncRNAs were present within one edge of protein-coding genes in cluster II that were associated with ECM (Fig. 6h). This sub-cluster is highly enriched in genes related to liver fibrosis, including 11 collagen genes and genes encoding TGFB1, matrix metalloproteinases, tissue inhibitors of metalloproteinases, and lysyl oxidase like proteins. These findings suggest that the 12 identity lncRNAs in this sub-cluster likely contribute to ECM production and liver fibrosis.

We then asked if coordinated expression of coding and noncoding genes within super-enhancers could explain the structure of the co-expression networks. Cluster I and cluster II contained 30 paired genes encoding lncRNAs and mRNAs that were co-expressed and located in the same super-enhancers, but these examples were almost entirely associated with divergently transcribed genes that were located in a super-enhancer. In contrast, cluster IV did contain two super-enhancers where multiple mRNAs and lncRNAs were co-expressed (Fig. 6i). While cluster IV does contain examples of coexpressed genes within super-enhancers, association within super-enhancers appears to account for only a small fraction of co-expressed genes.

The majority of IncRNAs expressed in HSC myofibroblasts are divergently transcribed from genes that encode proteins (Fig. 2a). We found that over $90 \%$ of divergent lncRNAs expressed in HSCs were paired with proteincoding genes that are also expressed in HSCs (Additional file 12: Table S11). We analyzed expression of each lncRNA and the paired protein-coding gene across 37 primary tissues [75] and the six tier 1 and tier 2 cell lines from the ENCODE project [92] and found that only $14 \%$ of these pairs are co-expressed across tissues (Spearman correlation $>0.7, p<4 \mathrm{e}-7$; Additional file 13: Table S12). Thus, in many cell types where a protein-coding gene is expressed, the divergent IncRNA identified in HSCs is silent.

\section{IncRNAs expressed in fetal HSCs exhibit expression patterns similar to those in adult HSCs}

The lncRNAs analyzed in this study were defined in human fetal HSCs myofibroblasts and we next asked if 
a
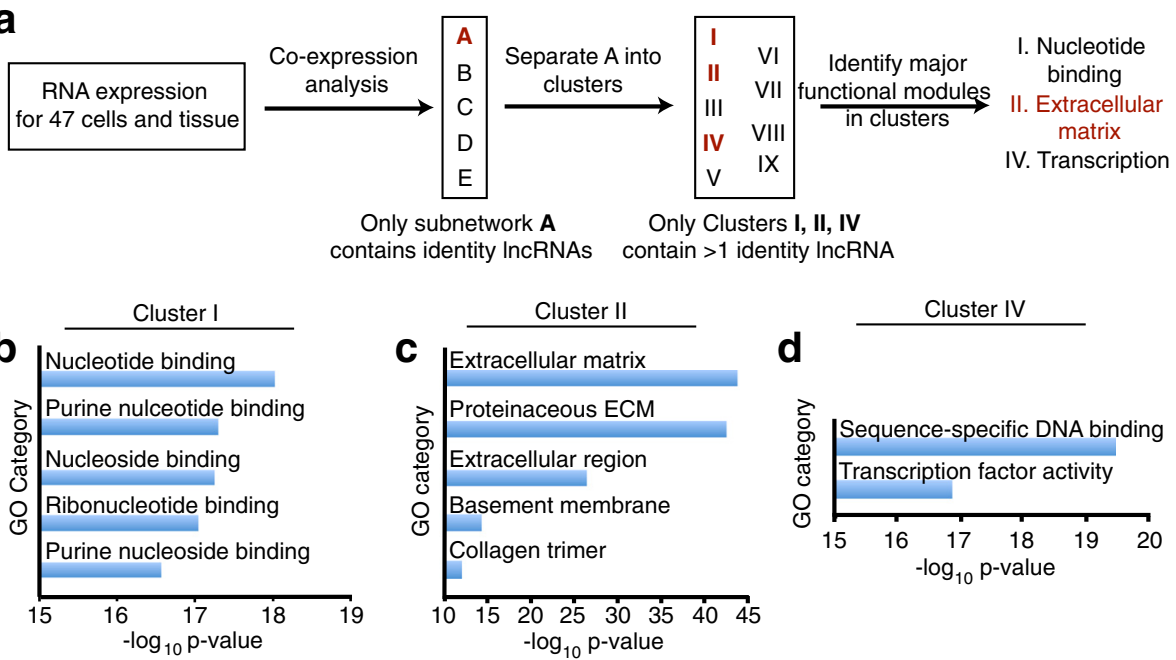

Only subnetwork A

Only Clusters I, II, IV contains identity IncRNAs contain $>1$ identity IncRNA
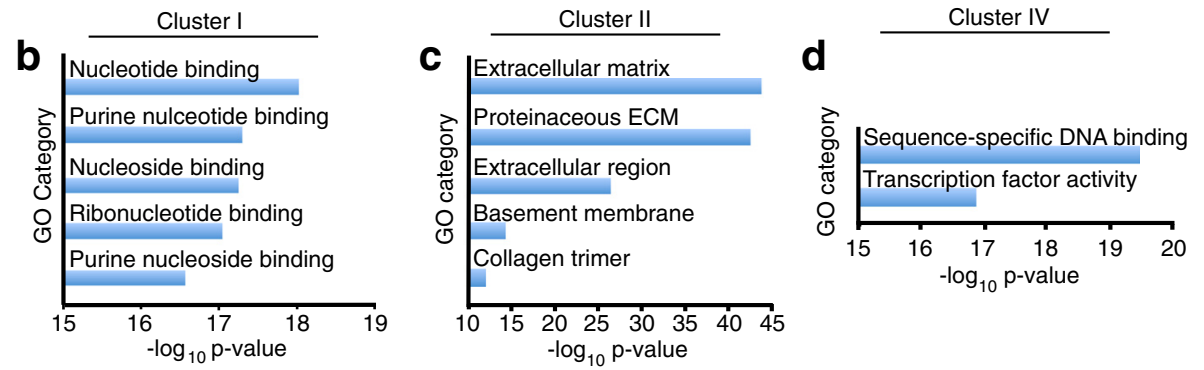

e
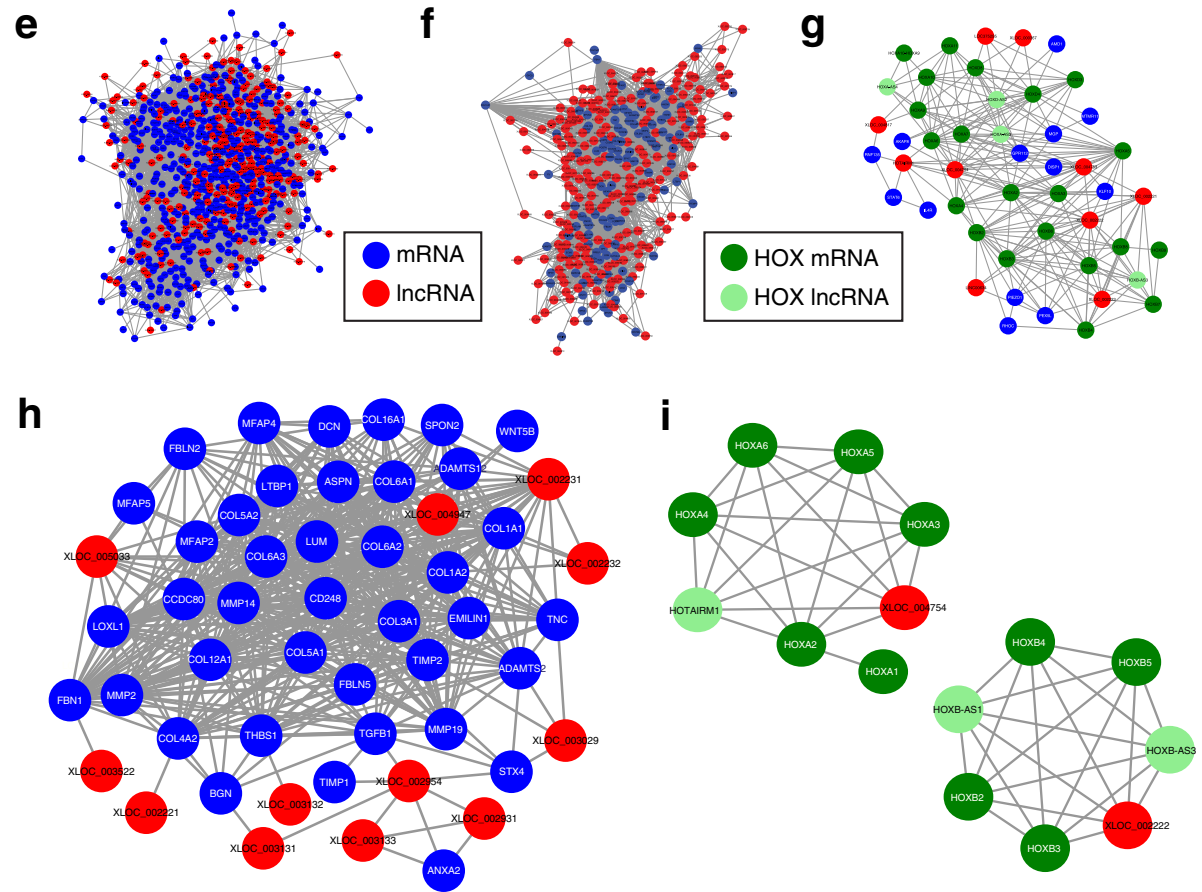

Fig. 6 IncRNAs discovered in HSCs form networks with ECM proteins. a Schematic of co-expression analysis. Expression of all protein-coding genes as well as the IncRNAs expressed in HSCs were analyzed in HSCs, 37 primary tissues, and six ENCODE cell lines. Co-expression analysis (Spearman coefficient $>0.7$ ) identified five main subnetworks. Only subnetwork A contained HSC identity IncRNAs. Subnetwork A was divided into nine main clusters. Only three clusters (red) contained more than one HSC identity IncRNA. Gene ontology (GO) analysis was performed to identify the most enriched categories for each of these three clusters. The extracellular matrix was significantly enriched in cluster II. The top GO categories identified for cluster I (b), cluster II (c), and cluster IV (d) are shown. The -log10 p values are displayed on the x-axis. e The protein-coding genes from cluster I that are contained in the GO category of nucleotide binding are displayed along with all HSC IncRNAs contained in cluster I that are connected to the nucleotide-binding protein-coding genes by one edge (gray lines). mRNAs (blue) and IncRNAs (red) are represented as circles. f The protein-coding genes from cluster II that are contained in the GO category of ECM are displayed along with all HSC IncRNAs contained in cluster II that are connected to the ECM proteins by one edge. $\mathbf{g}$ The entire network for cluster IV is displayed, including all protein-coding genes and HSC IncRNA genes. This cluster contains multiple HOX genes (dark green circles) and IncRNAs associated with HOX genes (HOX IncRNA, light green circles). $\mathbf{h}$ The HSC identity IncRNAs and the ECM proteins in cluster II that are connected by one edge are shown. HSC identity IncRNAs were defined as occupied by SMAD3, associated with super-enhancers, and restricted in expression to HSCs. $\mathbf{i}$ Two super-enhancers regulate genes encoding mRNAs and IncRNAs in cluster IV. Genes encoding mRNAs and IncRNAs located within a super-enhancer and connected by one edge are shown for two super-enhancers 
these findings also apply to adult HSC myofibroblasts. We performed RNA-sequencing to quantify expression of lncRNAs in primary human HSCs that were transdifferentiated into HSC myofibroblasts by ex vivo culture. Over 96 \% (3566 out of 3691) of lncRNAs detected in fetal HSC myofibroblasts were also detected in adult HSC myofibroblasts (Additional file 14: Table S13). The 435 lncRNAs that were uniquely enriched in fetal HSC myofibroblasts (Fig. 4) were also highly enriched in adult HSC myofibroblasts (Fig. 7a). We then asked if these lncRNAs were specific to HSC myofibroblasts or were also expressed in myofibroblasts originating in other tissues. We found that pancreatic stellate cells [103] and dermal fibroblasts [75] also expressed many lncRNAs in common with fetal HSC myofibroblasts (Additional file 3: Figure S7a, Additional file 14: Table S13). However, lncRNA expression in fetal HSC myofibroblasts was more closely associated with lncRNA expression in adult HSC myofibroblasts compared with pancreatic stellate cell myofibroblasts $(p<1.23$ e-7) or dermal fibroblasts $(p<8.8 \mathrm{e}-6)$ and is also shown by PCA (Fig. 7b). This association was also present when taking into account expression of both lncRNAs and protein-coding genes (Additional file 3: Figure S7b). In addition, adult HSC myofibroblasts stimulated with TGF- $\beta$ signaling showed similar patterns of gene induction and repression compared with fetal HSC myofibroblasts treated with TGF- $\beta$ (Additional file 3: Figure S7c, Additional file 15: Table S14).

Cluster II (Fig. 6f) contains the lncRNAs that form a network with ECM proteins. Adjusting this network to contain lncRNAs expressed in adult HSC myofibroblasts led to removal of only four lncRNAs (Additional file 3: Figure S7d). We then analyzed expression of the lncRNAs in the ECM module across normal human liver tissue, bridging fibrosis, and cirrhosis. This analysis identified 16 lncRNAs that were expressed in both fetal and adult HSC myofibroblasts and were significantly enriched in human liver fibrosis and cirrhosis compared with normal livers (Fig. 7c), suggesting that induction of these lncRNAs is associated with progression of human liver fibrosis. It also indicates that the ECM module is enriched in lncRNAs induced in bridging fibrosis and cirrhosis (7.6 \%) compared with the total lncRNA pool in HSCs (1.4\%).

A small fraction of the noncoding RNAs identified in HSCs can be classified as unidirectional eRNAs

lncRNAs were identified in this study by the presence of polyadenylation and size greater than $200 \mathrm{nt}$. eRNAs are noncoding RNAs (ncRNAs) transcribed from enhancers and tend not to be polyadenylated or spliced [104]. They can be transcribed unidirectionally or bidirectionally and unidirectional eRNAs can be polyadenylated [105]. The functional differences between polyadenylated unidirectional eRNAs and lncRNAs transcribed from enhancers are not clearly understood [106], but the abundance of the chromatin mark H3K4me1 compared with H3K4me3 has been used to classify these ncRNA transcripts $[22,80]$. To determine the fraction of IncRNAs identified in this study that overlap with polyadenylated unidirectional eRNAs, we analyzed ChIP-seq data of H3K4me1 and H3K4me3 in immortalized human induced fibroblasts (hiF-Ts) [107]. Of the 1042 lncRNAs identified in HSCs and classified as enhancer-associated or intergenic (Fig. 2a), 851 were also expressed in hiF-Ts. Using the H3K4me1:H3K4me3 ratio >1.2 as the threshold, we found that 181 of 851 ncRNAs ( $21 \%$ ) meet the definition of eRNAs (Fig. 7d; Additional file 16: Table S15) [80] and 90 of these loci encode only single exon ncRNAs. It is not clear if these unidirectional eRNAs may have different activities to lncRNAs, but eRNAs accounted for only $5.6 \%$ of lncRNAs in the ECM functional module (Fig. $6 f$ ) and removal of these eRNAs led to loss of two additional protein-coding genes from the ECM module (Additional file 3: Figure S7d and Additional file 11: Table S10).

\section{Discussion}

HSCs are the primary cell type responsible for liver fibrosis and liver failure in chronic liver disease. While many protein-coding genes that regulate HSC function have been described, the diversity of IncRNA expression in HSCs and the biological pathways they affect are unknown. This study was performed to define the lncRNAs expressed in human HSCs and to predict those that are likely to regulate the fibrotic process. Identification of IncRNAs uniquely enriched in HSCs will provide potential targets to inhibit the progression of fibrosis without affecting other cell types in the liver.

We also find that many lncRNAs are expressed divergently from protein-coding genes, as previously described $[55,58]$. While our analysis does not address co-expression of the paired protein-coding and lncRNA genes at the single cell level [108], it does suggest that the lncRNAs and their divergent protein-coding genes are usually both expressed is HSCs. In contrast, a minority of these paired lncRNA and protein-coding genes are co-expressed across different cell types. Thus, while a protein-coding gene may be expressed in many different cell types, the expression of its paired, divergent lncRNA appears to be more restricted, suggesting that there are cell type-specific levels of IncRNA gene regulation independent of the transcriptional control of the divergent protein-coding gene.

Our analysis also identified 195 lncRNAs that are directly affected by TGF- $\beta$ signaling as indicated by SMAD3 occupancy [90] and change in expression in response to TGF- $\beta$ signaling. In addition, lncRNAs directly regulated by TGF- $\beta$ signaling are enriched in super-enhancers, which suggests that the lncRNAs controlled by TGF- $\beta$ 


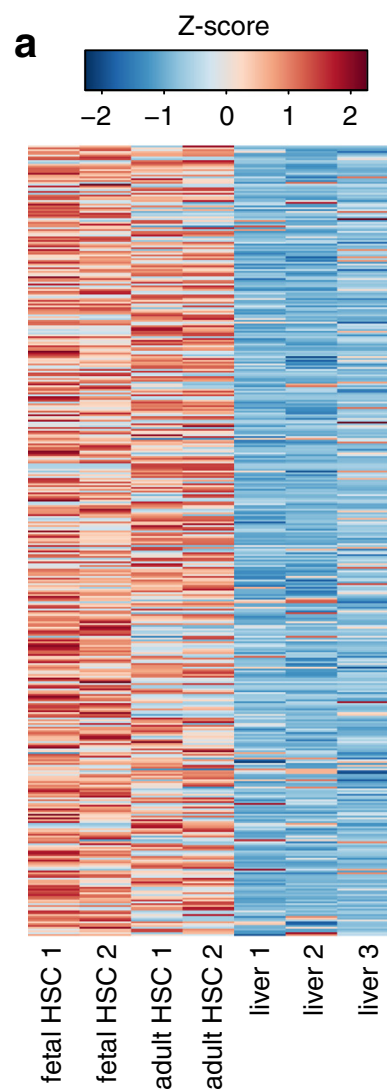

C

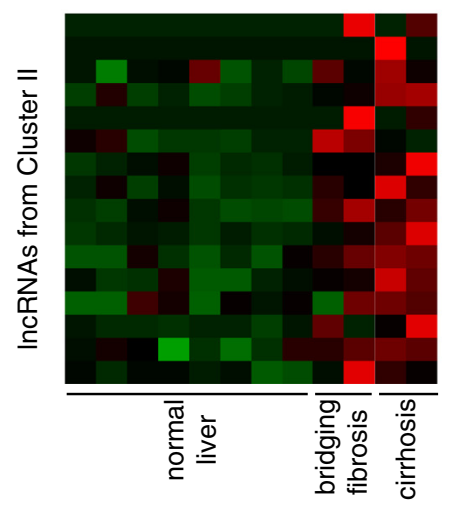

b
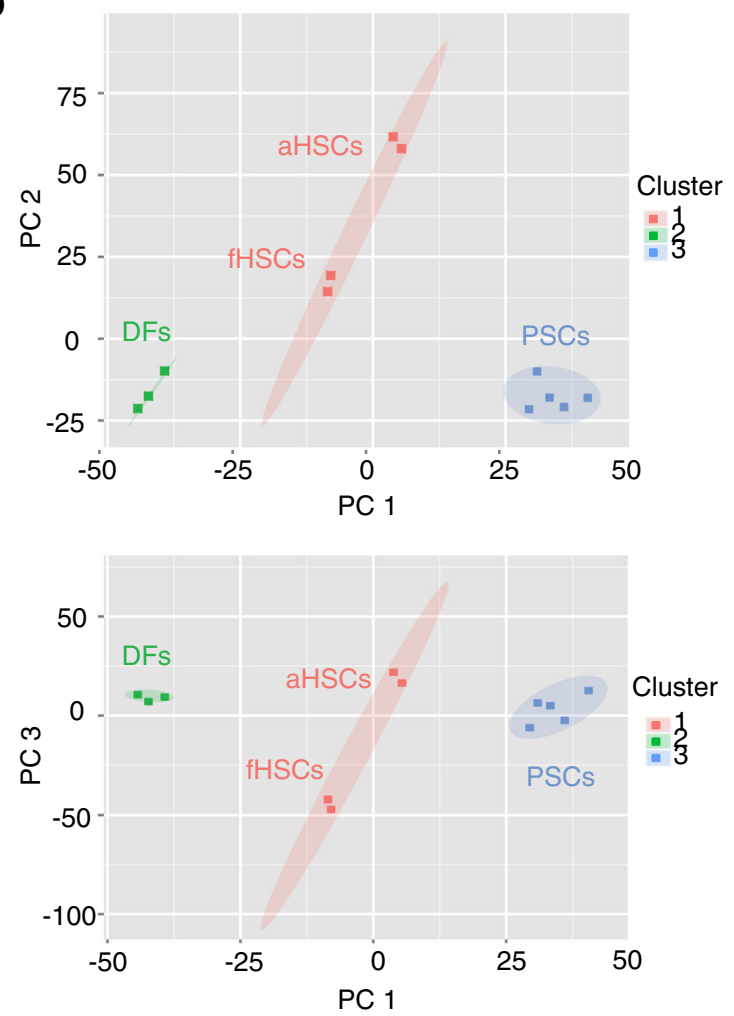

d

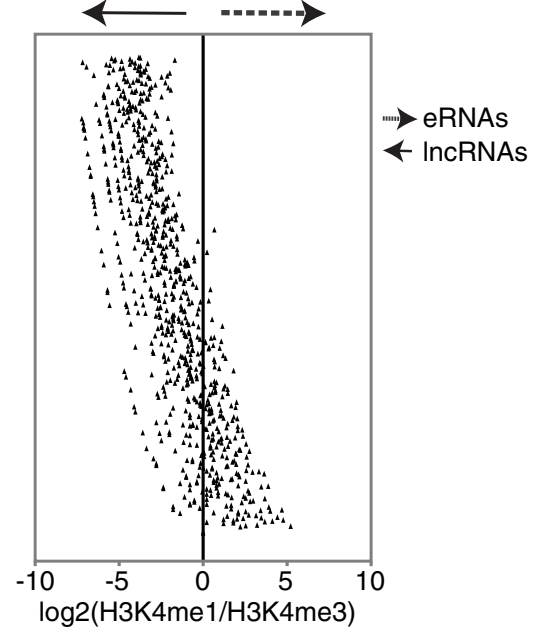

Fig. 7 IncRNA expression across myofibroblasts from different tissues. a Expression of HSC-specific IncRNAs (Fig. 4) was analyzed in fetal HSCS, adult HSCs, and liver. b PCA and k-mean clustering show that adult and fetal HSC myofibroblasts cluster together (aHSCs and fHSCS, red) compared with pancreatic stellate cell myofibroblasts (PSCs, blue) and dermal fibroblasts (DFs, green) based on IncRNA expressions. Principal component (PC)2 versus PC1 is displayed on the top and PC3 versus PC1 is displayed on the bottom. $\mathbf{c}$ Expression of IncRNAs contained in the ECM network (Fig. 6f) was analyzed across eight normal liver samples, two with bridging fibrosis and two with cirrhosis [75]. Sixteen IncRNAs from cluster II (y-axis) were found to be enriched in fibrosis and cirrhosis compared with normal livers. Each row represents expression of one IncRNA and the liver histology is indicated on the $x$-axis. The Z-score is shown in the upper right. $\mathbf{d}$ Identification of IncRNAs that meet criteria of unidirectional eRNAs. The ratio of H3K4me1/H3keme3 was calculated at the TSS $( \pm 0.5 \mathrm{~kb}$ ) of the selected IncRNAs. A ratio $>1.2$ was used to identify eRNAs (indicated by dotted arrow) [80] 
signaling may play key roles in HSC function and fibrosis. TGF- $\beta$ is a key activator of fibrosis [2] and this analysis was performed on HSC myofibroblasts that underwent transdifferentiation ex vivo as a model to identify human lncRNAs regulated by TGF- $\beta$ signaling. Now that these IncRNAs have been defined, it will be important to identify and characterize those that are induced in human liver disease.

Genes that control cell identity tend to be lineagerestricted [109-114]. To identify additional lncRNAs that may be key contributors to fibrosis progression, we defined the lncRNAs enriched in HSC myofibroblasts. We identified over 400 lncRNAs that are restricted in expression to HSCs compared with 43 other tissues and cell types. This analysis provides a more accurate representation of cell-type specificity than the identification of HSC IncRNAs that were not previously annotated because ab initio assembly of lncRNA transcripts has not been performed in all the tissues and cell types we were able to analyze. By defining the lncRNAs that were HSC-specific, bound by SMAD3, and associated with super-enhancers, we were able to focus on a small set of lncRNAs that are most likely to control HSC myofibroblast cell identity, which we refer to as identity lncRNAs. Tracing these lncRNAs through the co-expression network analysis allowed us to focus on the networks containing lncRNAs most relevant to HSC function.

Co-expression network analysis revealed that lncRNAs are co-expressed with protein-coding genes that regulate production of ECM proteins. Over half the genes in this network are lncRNAs and the network also includes numerous collagen genes, whose products make up the fibrotic scar, as well as proteins that are responsible for crosslinking and remodeling the ECM. Co-expression analysis was repeated in the absence of HSC expression data and confirmed that the ECM network is present. These findings indicate that the ECM network represents a robust co-expression network that is not identified solely based on high expression of genes in HSCs. In addition, a subset of the lncRNAs in the ECM network is induced in human liver fibrosis (Fig. 7c), suggesting that they are associated with human liver disease. HSCs make up only $5-10 \%$ of the cells in the liver [115] and additional lncRNAs are likely to be induced in HSCs during the progression of fibrosis that were not detected in whole liver samples due to lower levels of expression.

We found that lncRNA expression was highly conserved between fetal and adult HSC myofibroblasts. In addition, many lncRNAs were also shared between HSC myofibroblasts, pancreatic stellate cell myofibroblasts, and dermal fibroblasts. Analysis of IncRNAs alone or IncRNAs and protein-coding genes together revealed that fetal HSC myofibroblasts were more closely related to adult HSC myofibroblasts than to the other cell types analyzed. This study focused on the identification of lncRNAs in HSC myofibroblasts but also suggests that understanding the role of lncRNAs in HSC myofibroblasts may lead to insight into the function of lncRNAs in myofibroblasts from other tissues.

\section{Conclusions}

We provide the first comprehensive catalog of lncRNAs expressed in human HSCs and demonstrate that the lncRNAs identified are relevant to human liver disease. We discovered more than 3600 lncRNAs, including approximately $40 \%$ that have not been described in other cell types and greater than 400 that are uniquely enriched in HSCs compared with 43 other tissues and cell types. We analyzed the genomic location, chromatin modifications, response to differentiation and signaling, and expression across different tissues and cell types to identify lncRNAs that are likely to be involved in HSC function and fibrosis. This analysis provides a resource for future studies to investigate lncRNA function in liver disease and fibrosis.

\section{Additional files}

Additional file 1: Table S1. GTEx samples used in expression analyses. (XLS $40 \mathrm{~kb}$ )

Additional file 2: Table S2. GTEx ID and pathology reads of eight normal livers, two cirrhosis livers, and two bridging-fibrosis livers. (XLS $22 \mathrm{~kb}$ )

Additional file 3: Supplemental Figures and legends. (PDF 3744 kb) Additional file 4: Table S3. InCRNA transcripts and loci found in HSC myofibroblasts. (XLS 1520 kb)

Additional file 5: Table S4. The genomic locations of super-enhancers. (XLS $60 \mathrm{~kb}$ )

Additional file 6: Table S5. IncRNA transcripts and loci identified in HSC myofibroblasts, HSC myofibroblasts during serum starvation, and HSC myofibroblasts after TGF- $\beta$ stimulation. (XLS 3456 kb)

Additional file 7: Table S6. Expression levels of all IncRNA loci in each condition of HSC. (XLS 408 kb)

Additional file 8: Table S7. IncRNAs directly induced/repressed by TGF- $\beta$ signaling pathway. (XLSX 53 kb)

Additional file 9: Table S8. Expression profiles of HSC-specific IncRNAs across HSC myofibroblasts, TGF- $\beta$ HSCs, and 43 human tissues or cell types. (XLS $389 \mathrm{~kb}$ )

Additional file 10: Table S9. IncRNAs' association with SMAD3, superenhancers, and restricted expression in HSCs. (XLS $312 \mathrm{~kb}$ )

Additional file 11: Table S10. Co-expression pairs and gene lists of major functional modules in clusters I, II and IV. (XLS 988 kb)

Additional file 12: Table S11. Expression levels of IncRNAs and their divergent paired coding genes in HSC myofibroblasts. (XLS $310 \mathrm{~kb}$ )

Additional file 13: Table S12. Expression profile of divergently paired protein-coding genes and IncRNAs that are co-expressed across 43 human tissues and cell types. (XLSX $533 \mathrm{~kb}$ )

Additional file 14: Table S13. The mean expression levels in FPKM of IncRNAs in adult HSCs, PSCs, dermal fibroblasts, and livers. (XLSX 328 kb)

Additional file 15: Table S14. The expression levels in FPKM of IncRNAs in TGF- $\beta$ starvation and TGF- $\beta$ treatment adult HSCs. (XLSX $250 \mathrm{~kb}$ )

Additional file 16: Table S15. H3K4me1:H3K4me3 ratio of the 851 IncRNAs in hiF cells. (XLS $78 \mathrm{~kb}$ ) 


\section{Abbreviations}

ACTA2: actin, alpha2 smooth muscle; ChIP: chromatin immunoprecipitation: DMEM: Dulbecco's modified Eagle medium; ECM: extracellular matrix; ENCODE: Encyclopedia of DNA Elements; eRNA: enhancer RNA; FCS: fetal calf serum; FPKM: fragments per kilobase of transcript per million fragments mapped; GEO: Gene Expression Omnibus; GO: Gene Ontology; GTEx: Genotype-Tissue Expression; HCC: hepatocellular carcinoma hiF-T: human induced fibroblast; HSC: hepatic stellate cell; HUVEC: human umbilical vein endothelial cell; kb: kilobase; IncRNA: long noncoding RNA logLR: log-likelihood ratio; LOX: lysyl oxidase; LOXL2: Iysyl oxidase like 2; MCL: Markov Clustering; mRNA: messenger RNA; ncRNA: noncoding RNA nt: nucleotide; P/S: penicillin/streptomycin; PC: principal component; PCA: principal component analysis; snoRNA: small nucleolar RNA; TGF- $\beta$ : transforming growth factor beta; TSS: transcription start site; UCSC: University of California, Santa Cruz.

\section{Competing interests}

The authors declare that they have no competing interests.

\section{Authors' contributions}

The project was conceived and directed by CZ and ACM. Data analysis was performed by CZ with assistance from JVP and DLM. Cell culture work was performed by SRY and JYC. Libraries were prepared for RNA sequencing by SRY and JVP and microscopy was performed by JYC. The manuscript was written by CZ and ACM with input from RTC. All authors read and approved the final manuscript

\section{Acknowledgements}

We thank Kaveh Daneshvar for helpful discussions and assistance with image processing and Scott Friedman for helpful discussions and review of the manuscript. We also thank Igor Ulitsky for advice on determining proteincoding potential. We thank Massachusetts General Hospital (MGH) Next Generation Sequencing Core for sequencing and the MGH Program in Membrane Biology microscopy core. This project was supported by NIDDK DK078772 and DK098079 (RTC) and the American Gastroenterological Association Elsevier Pilot Award and Massachusetts General Hospital Department of Medicine Transformative Scholar Award (ACM).

\section{Received: 22 January 2016 Accepted: 3 March 2016}

\section{Published online: 23 March 2016}

\section{References}

1. Bataller R, Brenner DA. Liver fibrosis. J Clin Invest. 2005:115:209-18.

2. Friedman SL. Hepatic stellate cells: protean, multifunctional, and enigmatic cells of the liver. Physiol Rev. 2008;88:125-72.

3. Rojkind M, Martinez-Palomo A. Increase in type I and type III collagens in human alcoholic liver cirrhosis. Proc Natl Acad Sci U S A. 1976;73:539-43.

4. Rojkind M, Giambrone M-A, Biempica L. Collagen types in normal and cirrhotic liver. Gastroenterology. 1979;76:710-9.

5. Clement B, Grimaud J-A, Campion J-P, Deugnier Y, Guillouzo A. Cell types involved in collagen and fibronectin production in normal and fibrotic human liver. Hepatology. 1986;6:225-34.

6. Hahn E, Wick G, Pencev D, Timpl R. Distribution of basement membrane proteins in normal and fibrotic human liver: collagen type IV, laminin, and fibronectin. Gut. 1980;21:63-71.

7. Sanderson N, Factor V, Nagy P, Kopp J, Kondaiah P, Wakefield L, et al. Hepatic expression of mature transforming growth factor beta 1 in transgenic mice results in multiple tissue lesions. Proc Natl Acad Sci U S A. 1995:92:2572-6.

8. Clouthier DE, Comerford SA, Hammer RE. Hepatic fibrosis, glomerulosclerosis, and a lipodystrophy-like syndrome in PEPCK-TGF-beta1 transgenic mice. J Clin Invest. 1997;100:2697-713.

9. Friedman SL, Roll FJ, Boylest J, Bissell DM. Hepatic lipocytes: the principal collagen-producing cells of normal rat liver. Proc Natl Acad Sci U S A. 1985;82:8681-5.

10. Mederacke I, Hsu CC, Troeger JS, Huebener P, Mu X, Dapito DH, et al. Fate tracing reveals hepatic stellate cells as dominant contributors to liver fibrosis independent of its aetiology. Nat Commun. 2013;4:2823.

11. Friedman SL, Rockey DC, McGuire RF, Maher JJ, Boyles JK, Yamasaki G. Isolated hepatic lipocytes and Kupffer cells from normal human liver: morphological and functional characteristics in primary culture. Hepatology. 1992;15:234-43.

12. Uemura M, Swenson ES, Gaça MDA, Giordano FJ, Reiss M, Wells RG. Smad2 and Smad3 play different roles in rat hepatic stellate cell function and alpha-smooth muscle actin organization. Mol Biol Cell. 2005;16:4214-24.

13. Kisseleva T, Cong M, Paik Y, Scholten D, Jiang C, Benner C, et al. Myofibroblasts revert to an inactive phenotype during regression of liver fibrosis. Proc Natl Acad Sci U S A. 2012;109:9448-53.

14. Troeger JS, Mederacke I, Gwak G-Y, Dapito DH, Mu X, Hsu CC, et al. Deactivation of hepatic stellate cells during liver fibrosis resolution in mice. Gastroenterology. 2012;143:1073-83. e22.

15. Hellerbrand C, Stefanovic B, Giordano F, Burchardt ER, Brenner DA. The role of TGF $\beta 1$ in initiating hepatic stellate cell activation in vivo. J Hepatol. 1999:30:77-87.

16. Kanzler S, Lohse AW, Keil A, Henninger J, Dienes HP, Schirmacher P, et al. TGF-beta 1 in liver fibrosis: an inducible transgenic mouse model to study liver fibrogenesis. Am J Physiol Gastrointest Liver Physiol. 1999:276:G1059-1068.

17. Ueberham E, Löw R, Ueberham U, Schönig K, Bujard H, Gebhardt R. Conditional tetracycline-regulated expression of TGF-beta1 in liver of transgenic mice leads to reversible intermediary fibrosis. Hepatology. 2003;37:1067-78.

18. Schmitt-Gräff A, Krüger S, Bochard F, Gabbiani G, Denk H. Modulation of alpha smooth muscle actin and desmin expression in perisinusoidal cells of normal and diseased human livers. Am J Pathol. 1991;138:1233-42.

19. Rockey DC, Boyles JK, Gabbiani G, Friedman SL. Rat hepatic lipocytes express smooth muscle actin upon activation in vivo and in culture. J Submicrosc Cytol Pathol. 1992;24:193-203.

20. Perepelyuk M, Terajima M, Wang AY, Georges PC, Janmey PA, Yamauchi M, et al. Hepatic stellate cells and portal fibroblasts are the major cellular sources of collagens and lysyl oxidases in normal liver and early after injury. Am J Physiol Gastrointest Liver Physiol. 2013;304:G605-14.

21. Liu X, Xu J, Brenner DA, Kisseleva T. Reversibility of liver fibrosis and inactivation of fibrogenic myofibroblasts. Curr Pathobiol Rep. 2013;1:209-14.

22. Djebali S, Davis CA, Merkel A, Dobin A, Lassmann T, Mortazavi A, et al. Landscape of transcription in human cells. Nature. 2012;489:101-8.

23. Rinn JL, Chang HY. Genome regulation by long noncoding RNAs. Annu Rev Biochem. 2012:81:145-66.

24. Xie C, Yuan J, Li H, Li M, Zhao G, Bu D, et al. NONCODEv4: exploring the world of long non-coding RNA genes. Nucleic Acids Res. 2014;42(Database issue):D98-103.

25. Brown CJ, Lafreniere RG, Powers VE, Sebastio G, Ballabio A, Pettigrew AL, et al. Localization of the $X$ inactivation centre on the human $X$ chromosome in Xq13. Nature. 1991;349:82-4.

26. Zhao J, Sun BK, Erwin JA, Song J-J, Lee JT. Polycomb proteins targeted by a short repeat RNA to the mouse X chromosome. Science. 2008;322:750-6.

27. Khalil AM, Guttman M, Huarte M, Garber M, Raj A, Rivea Morales D, et al. Many human large intergenic noncoding RNAs associate with chromatinmodifying complexes and affect gene expression. Proc Natl Acad Sci U S A. 2009;106:11667-72.

28. Kretz M, Siprashvili Z, Chu C, Webster DE, Zehnder A, Qu K, et al. Control of somatic tissue differentiation by the long non-coding RNA TINCR. Nature. 2013:493:231-5.

29. Yoon J-H, Abdelmohsen K, Kim J, Yang X, Martindale JL, TominagaYamanaka $\mathrm{K}$, et al. Scaffold function of long non-coding RNA HOTAIR in protein ubiquitination. Nat Commun. 2013:4:2939.

30. Wang $P$, Xue $Y$, Han $Y$, Lin L, Wu C, Xu S, et al. The STAT3-binding long noncoding RNA Inc-DC controls human dendritic cell differentiation. Science. 2014;344:310-3.

31. Li L, Liu B, Wapinski OL, Tsai M-C, Qu K, Zhang J, et al. Targeted disruption of Hotair leads to homeotic transformation and gene derepression. Cell Rep. 2013:5:3-12.

32. Sauvageau M, Goff LA, Lodato S, Bonev B, Groff AF, Gerhardinger C, et al. Multiple knockout mouse models reveal lincRNAs are required for life and brain development. Elife. 2013;2:1-24

33. Klattenhoff CA, Scheuermann JC, Surface LE, Bradley RK, Fields PA, Steinhauser ML, et al. Braveheart, a long noncoding RNA required for cardiovascular lineage commitment. Cell. 2013;152:570-83.

34. Herriges MJ, Swarr DT, Morley MP, Rathi KS, Peng T, Stewart KM, et al. Long noncoding RNAs are spatially correlated with transcription factors and regulate lung development. Genes Dev. 2014;28:1363-79. 
35. Carpenter S, Aiello D, Atianand MK, Ricci EP, Gandhi P, Hall LL, et al. A long noncoding RNA mediates both activation and repression of immune response genes. Science. 2013;341:789-92.

36. Han P, Li W, Lin C-H, Yang J, Shang C, Nuernberg ST, et al. A long noncoding RNA protects the heart from pathological hypertrophy. Nature. 2014:514:102-6.

37. Du Z, Fei T, Verhaak RGW, Su Z, Zhang Y, Brown M, et al. Integrative genomic analyses reveal clinically relevant long noncoding RNAs in human cancer. Nat Struct Mol Biol. 2013;20:908-13.

38. Ponjavic J, Oliver PL, Lunter G, Ponting CP. Genomic and transcriptional co-localization of protein-coding and long non-coding RNA pairs in the developing brain. PLoS Genet. 2009;5:e1000617.

39. Panzitt K, Tschernatsch MMO, Guelly C, Moustafa T, Stradner M, Strohmaier HM, et al. Characterization of HULC, a novel gene with striking up-regulation in hepatocellular carcinoma, as noncoding RNA. Gastroenterology. 2007;132:330-42.

40. Lin R, Maeda S, Liu C, Karin M, Edgington TS. A large noncoding RNA is a marker for murine hepatocellular carcinomas and a spectrum of human carcinomas. Oncogene. 2007;26:851-8.

41. Braconi C, Valeri N, Kogure T, Gasparini P, Huang N, Nuovo GJ, et al. Expression and functional role of a transcribed noncoding RNA with an ultraconserved element in hepatocellular carcinoma. Proc Natl Acad Sci U S A. 2011;108:786-91.

42. Yang F, Zhang L, Huo X, Yuan J, Xu D, Yuan S, et al. Long noncoding RNA high expression in hepatocellular carcinoma facilitates tumor growth through enhancer of zeste homolog 2 in humans. Hepatology. 2011:54:1679-89.

43. Yang Z, Zhou L, Wu L-M, Lai M-C, Xie H-Y, Zhang F, et al. Overexpression of long non-coding RNA HOTAIR predicts tumor recurrence in hepatocellular carcinoma patients following liver transplantation. Ann Surg Oncol. 2011;18:1243-50.

44. Yuan S-X, Yang F, Yang Y, Tao Q-F, Zhang J, Huang G, et al. Long noncoding RNA associated with microvascular invasion in hepatocellular carcinoma promotes angiogenesis and serves as a predictor for hepatocellular carcinoma patients' poor recurrence-free survival after hepatectomy. Hepatology. 2012:56:2231-41.

45. Takahashi K, Yan IK, Haga H, Patel T. Modulation of hypoxia-signaling pathways by extracellular linc-RoR. J Cell Sci. 2014;127:1585-94.

46. Quagliata L, Matter MS, Piscuoglio S, Arabi L, Ruiz C, Procino A, et al. Long noncoding RNA HOTTIP/HOXA13 expression is associated with disease progression and predicts outcome in hepatocellular carcinoma patients. Hepatology. 2014;59:911-23.

47. Tsang FHC, Au SLK, Wei L, Fan DNY, Lee JMF, Wong CCL, et al. Long noncoding RNA HOTTIP is frequently up-regulated in hepatocellular carcinoma and is targeted by tumour suppressive miR-125b. Liver Int. 2015;35:1597-606.

48. Mann DA. Epigenetics in liver disease. Hepatology. 2014;60:1418-25.

49. Lai M, Yang Z, Zhou L, Zhu Q, Xie H, Zhang F, et al. Long non-coding RNA MALAT-1 overexpression predicts tumor recurrence of hepatocellular carcinoma after liver transplantation. Med Oncol. 2012;29:1810-6.

50. Kogure T, Yan IK, Lin W-L, Patel T. Extracellular vesicle-mediated transfer of a novel long noncoding RNA TUC339: a mechanism of intercellular signaling in human hepatocellular cancer. Genes Cancer. 2013;4:261-72.

51. Xu D, Yang F, Yuan J, Zhang L, Bi H, Zhou C, et al. Long noncoding RNAs associated with liver regeneration 1 accelerates hepatocyte proliferation during liver regeneration by activating Wnt/ $\beta$-catenin signaling. Hepatology. 2013;58:739-51

52. He Y, Wu Y-T, Huang C, Meng X-M, Ma T-T, Wu B-M, et al. Inhibitory effects of long noncoding RNA MEG3 on hepatic stellate cells activation and liver fibrogenesis. Biochim Biophys Acta. 2014;1842(11):2204-15.

53. Yu F, Zheng J, Mao Y, Dong P, Lu Z, Li G, et al. Long non-coding RNA growth arrest-specific transcript 5 (GAS5) inhibits liver fibrogenesis through a mechanism of competing endogenous RNA. J Biol Chem. 2015;290:28286-98.

54. Braconi C, Kogure T, Valeri N, Huang N, Nuovo G, Costinean S, et al. microRNA-29 can regulate expression of the long non-coding RNA gene MEG3 in hepatocellular cancer. Oncogene. 2011;30:4750-6.

55. Cabili MN, Trapnell C, Goff L, Koziol M, Tazon-Vega B, Regev A, et al. Integrative annotation of human large intergenic noncoding RNAs reveals global properties and specific subclasses. Genes Dev. 2011;25:1915-27.

56. Derrien T, Johnson R, Bussotti G, Tanzer A, Djebali S, Tilgner H, et al. The GENCODE $\mathrm{V} 7$ catalog of human long noncoding RNAs: analysis of their gene structure, evolution, and expression. Genome Res. 2012;22:1775-89.
57. Hangauer MJ, Vaughn IW, McManus MT. Pervasive transcription of the human genome produces thousands of previously unidentified long intergenic noncoding RNAs. PLoS Genet. 2013;9:e1003569.

58. Sigova AA, Mullen AC, Molinie B, Gupta S, Orlando DA, Guenther MG. Divergent transcription of long noncoding RNA/mRNA gene pairs in embryonic stem cells. Proc Natl Acad Sci U S A. 2013;110:2876-81.

59. Trapnell C, Pachter L, Salzberg SL. TopHat: discovering splice junctions with RNA-Seq. Bioinformatics. 2009;25:1105-11.

60. Trapnell C, Roberts A, Goff L, Pertea G, Kim D, Kelley DR, et al. Differential gene and transcript expression analysis of RNA-seq experiments with TopHat and Cufflinks. Nat Protoc. 2012;7:562-78.

61. Trapnell C, Williams BA, Pertea G, Mortazavi A, Kwan G, van Baren MJ, et al. Transcript assembly and quantification by RNA-Seq reveals unannotated transcripts and isoform switching during cell differentiation. Nat Biotechnol. 2010;28:511-5.

62. Guttman M, Garber M, Levin JZ, Donaghey J, Robinson J, Adiconis X, et al. Ab initio reconstruction of cell type-specific transcriptomes in mouse reveals the conserved multi-exonic structure of lincRNAs. Nat Biotechnol. 2010;28:503-10.

63. Kent WJ, Sugnet CW, Furey TS, Roskin KM, Pringle TH, Zahler AM, et al. The Human Genome Browser at UCSC. Genome Res. 2002;12:996-1006.

64. Eddy SR. A new generation of homology search tools based on probabilistic inference. Genome Inform. 2009;23:205-11.

65. Finn RD, Clements J, Eddy SR. HMMER web server: interactive sequence similarity searching. Nucleic Acids Res. 2011;39(Web Server issue):W29-37.

66. Wang L, Park HJ, Dasari S, Wang S, Kocher J-P, Li W. CPAT: Coding-potential assessment tool using an alignment-free logistic regression model. Nucleic Acids Res. 2013;41:e74.

67. Finn RD, Bateman A, Clements J, Coggill P, Eberhardt RY, Eddy SR, et al. Pfam: the protein families database. Nucleic Acids Res. 2014:42(Database issue):D222-30.

68. Langmead B, Salzberg SL. Fast gapped-read alignment with Bowtie 2. Nat Methods. 2012;9:357-9.

69. Zhang Y, Liu T, Meyer CA, Eeckhoute J, Johnson DS, Bernstein BE, et al. Model-based analysis of ChIP-Seq (MACS). Genome Biol. 2008;9:R137.

70. Whyte WA, Orlando DA, Hnisz D, Abraham BJ, Lin CY, Kagey MH, et al. Master transcription factors and mediator establish super-enhancers at key cell identity genes. Cell. 2013;153:307-19.

71. Hnisz D, Abraham BJ, Lee TI, Lau A, Saint-André V, Sigova AA, et al. Superenhancers in the control of cell identity and disease. Cell. 2013;155:934-47.

72. Mercer TR, Gerhardt DJ, Dinger ME, Crawford J, Trapnell C, Jeddeloh JA, et al. Targeted RNA sequencing reveals the deep complexity of the human transcriptome. Nat Biotechnol. 2012;30:99-104.

73. Anders S, Pyl PT, Huber W. HTSeq-a Python framework to work with highthroughput sequencing data. Bioinformatics. 2014;31:166-9.

74. Love MI, Huber W, Anders S. Moderated estimation of fold change and dispersion for RNA-seq data with DESeq2. Genome Biol. 2014;15:550.

75. Lonsdale J, Thomas J, Salvatore M, Phillips R, Lo E, Shad S, et al. The Genotype-Tissue Expression (GTEx) project. Nat Genet. 2013;45:580-5.

76. van Dongen S, Abreu-Goodger C. Using MCL to extract clusters from networks. Methods Mol Biol. 2012;804:281-95.

77. Cline MS, Smoot M, Cerami E, Kuchinsky A, Landys N, Workman C, et al. Integration of biological networks and gene expression data using Cytoscape. Nat Protoc. 2007:2:2366-82.

78. Huang DW, Sherman BT, Lempicki RA. Systematic and integrative analysis of large gene lists using DAVID bioinformatics resources. Nat Protoc. 2009;4:44-57

79. Jiao X, Sherman BT, Huang DW, Stephens R, Baseler MW, Lane HC, et al. DAVID-WS: a stateful web service to facilitate gene/protein list analysis. Bioinformatics. 2012;28:1805-6.

80. Ilott NE, Heward JA, Roux B, Tsitsiou E, Fenwick PS, Lenzi L, et al. Long non-coding RNAs and enhancer RNAs regulate the lipopolysaccharideinduced inflammatory response in human monocytes. Nat Commun. 2014,5:3979.

81. Friedman SL, Roll FJ, Boyles J, Arenson DM, Bissell DM. Maintenance of differentiated phenotype of cultured rat hepatic lipocytes by basement membrane matrix. J Biol Chem. 1989;264:10756-62.

82. Sohara N, Znoyko I, Levy MT, Trojanowska M, Reuben A. Reversal of activation of human myofibroblast-like cells by culture on a basement membrane-like substrate. J Hepatol. 2002;37:214-21.

83. Ilott NE, Ponting CP. Predicting long non-coding RNAs using RNA sequencing. Methods. 2013;63:50-9. 
84. Pruitt KD, Brown GR, Hiatt SM, Thibaud-Nissen F, Astashyn A, Ermolaeva O, et al. RefSeq: an update on mammalian reference sequences. Nucleic Acids Res. 2014;42(Database issue):D756-63.

85. Karolchik D, Hinrichs AS, Furey TS, Roskin KM, Sugnet CW, Haussler D, et al. The UCSC Table Browser data retrieval tool. Nucleic Acids Res. 2004;32(Database issue):D493-6.

86. Guenther MG, Levine SS, Boyer LA, Jaenisch R, Young RA. A chromatin landmark and transcription initiation at most promoters in human cells. Cell. 2007;130:77-88.

87. Creyghton MP, Cheng AW, Welstead GG, Kooistra T, Carey BW, Steine EJ et al. Histone H3K27ac separates active from poised enhancers and predicts developmental state. Proc Natl Acad Sci U S A. 2010;107:21931-6.

88. Pauli A, Valen E, Lin MF, Garber M, Vastenhouw NL, Levin JZ, et al. Systematic identification of long noncoding RNAs expressed during zebrafish embryogenesis. Genome Res. 2012;22:577-91.

89. Massagué J, Seoane J, Wotton D. Smad transcription factors. Genes Dev. 2005:19:2783-810

90. Ding N, Yu RT, Subramaniam N, Sherman MH, Wilson C, Rao R, et al. A vitamin $D$ receptor/SMAD genomic circuit gates hepatic fibrotic response. Cell. 2013;153:601-13.

91. Mullen AC, Orlando DA, Newman JJ, Lovén J, Kumar RM, Bilodeau S, et al. Master transcription factors determine cell-type-specific responses to TGF- $\beta$ signaling. Cell. 2011;147:565-76

92. Consortium TEP. A user's guide to the encyclopedia of DNA elements (ENCODE). PLoS Biol. 2011:9:e1001046.

93. Luo F, Yang Y, Zhong J, Gao H, Khan L, Thompson DK, et al. Constructing gene co-expression networks and predicting functions of unknown genes by random matrix theory. BMC Bioinformatics. 2007:8:299.

94. Sharan R, Ulitsky I, Shamir R. Network-based prediction of protein function. Mol Syst Biol. 2007;3:88.

95. Wren JD. A global meta-analysis of microarray expression data to predict unknown gene functions and estimate the literature-data divide. Bioinformatics. 2009;25:1694-701.

96. Zhou $C$, Yin $Y$, Dam $P, X u$ Y. Identification of novel proteins involved in plant cell-wall synthesis based on protein-protein interaction data. J Proteome Res. 2010;9:5025-37.

97. Liao Q, Liu C, Yuan X, Kang S, Miao R, Xiao H, et al. Large-scale prediction of long non-coding RNA functions in a coding-non-coding gene co-expression network. Nucleic Acids Res. 2011;39:3864-78.

98. Ruan J, Dean AK, Zhang W. A general co-expression network-based approach to gene expression analysis: comparison and applications. BMC Systems Biol. 2010;4:8

99. Guo X, Gao L, Liao Q, Xiao H, Ma X, Yang X, et al. Long non-coding RNAs function annotation: a global prediction method based on bi-colored networks. Nucleic Acids Res. 2013;41:e35.

100. Xiao X, Moreno-Moral A, Rotival M, Bottolo L, Petretto E. Multi-tissue analysis of co-expression networks by higher-order generalized singular value decomposition identifies functionally coherent transcriptional modules. PLoS Genet. 2014;10:e1004006.

101. Lee HK, Hsu AK, Sajdak J, Qin J, Pavlidis P. Coexpression analysis of human genes across many microarray data sets. Genome Res. 2004;14:1085-94.

102. Eisen MB, Spellman PT, Brown PO, Botstein D. Cluster analysis and display of genome-wide expression patterns. Proc Natl Acad Sci U S A. 1998;95:14863-8.

103. Sherman $M H$, Yu RT, Engle DD, Ding N, Atkins AR, Tiriac $H$, et al. Vitamin D receptor-mediated stromal reprogramming suppresses pancreatitis and enhances pancreatic cancer therapy. Cell. 2014;159:80-93.

104. Kim T-K, Hemberg M, Gray JM, Costa AM, Bear DM, Wu J, et al. Widespread transcription at neuronal activity-regulated enhancers. Nature. 2010:465:182-7.

105. Koch F, Fenouil R, Gut M, Cauchy P, Albert TK, Zacarias-Cabeza J, et al. Transcription initiation platforms and GTF recruitment at tissue-specific enhancers and promoters. Nat Struct Mol Biol. 2011;18:956-63.

106. Lam MTY, Li W, Rosenfeld MG, Glass CK. Enhancer RNAs and regulated transcriptional programs. Trends Biochem Sci. 2014;39:170-82.

107. Cacchiarelli D, Trapnell C, Ziller MJ, Soumillon M, Cesana M, Karnik R, et al. Integrative analyses of human reprogramming reveal dynamic nature of induced pluripotency. Cell. 2015;162:412-24.
108. Cabili MN, Dunagin MC, McClanahan PD, Biaesch A, Padovan-Merhar O, Regev A, et al. Localization and abundance analysis of human IncRNAs at single-cell and single-molecule resolution. Genome Biol. 2015;16:20.

109. Lassar AB, Paterson BM, Weintraub $H$. Transfection of a DNA locus that mediates the conversion of $10 \mathrm{~T} 1 / 2$ fibroblasts to myoblasts. Cell. 1986;47:649-56.

110. Kulessa H, Frampton J, Graf T. GATA-1 reprograms avian myelomonocytic cell lines into eosinophils, thromboblasts, and erythroblasts. Genes Dev. 1995;9:1250-62

111. Feng R, Desbordes SC, Xie H, Tillo ES, Pixley F, Stanley ER, et al. PU.1 and C/EBPalpha/beta convert fibroblasts into macrophage-like cells. Proc Natl Acad Sci U S A. 2008;105:6057-62.

112. Takahashi K, Yamanaka S. Induction of pluripotent stem cells from mouse embryonic and adult fibroblast cultures by defined factors. Cell. 2006:126:663-76.

113. Seale P, Bjork B, Yang W, Kajimura S, Chin S, Kuang S, et al. PRDM16 controls a brown fat/skeletal muscle switch. Nature. 2008:454:961-7.

114. Zhou Q, Brown J, Kanarek A, Rajagopal J, Melton DA. In vivo reprogramming of adult pancreatic exocrine cells to beta-cells. Nature. 2008;455:627-32.

115. Knook D, Seffelaar A, de Leeuw AM. Fat-storing cells of the rat liver. Their isolation and purification. Exp Cell Res. 1982;139:468-71.

\section{Submit your next manuscript to BioMed Central and we will help you at every step:}

- We accept pre-submission inquiries

- Our selector tool helps you to find the most relevant journal

- We provide round the clock customer support

- Convenient online submission

- Thorough peer review

- Inclusion in PubMed and all major indexing services

- Maximum visibility for your research

Submit your manuscript at www.biomedcentral.com/submit 